ARTICLE

https://doi.org/10.1038/s41467-020-16211-x

\title{
Legionella effector MavC targets the Ube2N Ub conjugate for noncanonical ubiquitination
}

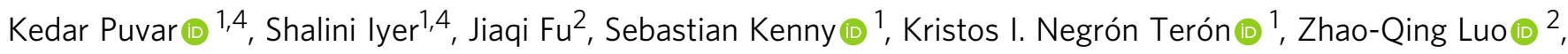
Peter S. Brzovic ${ }^{3,4}$, Rachel E. Klevit ${ }^{3 凶} \&$ Chittaranjan Das (i) ${ }^{1 凶}$

The bacterial effector MavC modulates the host immune response by blocking Ube2N activity employing an E1-independent ubiquitin ligation, catalyzing formation of a $\gamma$-glutamyl$\varepsilon$-Lys ( $\mathrm{Gln} 40^{\mathrm{Ub}}$-Lys92 $\mathrm{Ube} 2 \mathrm{~N}$ ) isopeptide crosslink using a transglutaminase mechanism. Here we provide biochemical evidence in support of MavC targeting the activated, thioester-linked Ube2N ubiquitin conjugate, catalyzing an intramolecular transglutamination reaction, covalently crosslinking the Ube2N and Ub subunits effectively inactivating the E2 Ub conjugate. Ubiquitin exhibits weak binding to $\mathrm{MavC}$ alone, but shows an increase in affinity when tethered to Ube2N in a disulfide-linked substrate that mimics the charged E2 Ub conjugate. Crystal structures of MavC in complex with the substrate mimic and crosslinked product provide insights into the reaction mechanism and underlying protein dynamics that favor transamidation over deamidation, while revealing a crucial role for the structurally unique insertion domain in substrate recognition. This work provides a structural basis of ubiquitination by transglutamination and identifies this enzyme's true physiological substrate.

\footnotetext{
${ }^{1}$ Department of Chemistry, Purdue University, West Lafayette, IN 47907, USA. ${ }^{2}$ Purdue Institute for Inflammation, Immunology and Infectious Disease and Department of Biological Sciences, Purdue University, West Lafayette, IN 47907, USA. ${ }^{3}$ Department of Biochemistry, University of Washington, Seattle, WA 98195, USA. ${ }^{4}$ These authors contributed equally: Kedar Puvar, Shalini lyer, Peter S. Brzovic. ${ }^{凶}$ email: klevit@uw.edu; cdas@purdue.edu
} 
$\mathrm{P}$ rotein ubiquitination is a post-translational modification used by eukaryotic organisms to regulate critical cellular processes such as protein quality control, cell cycle progression, DNA repair, autophagy, and immunity ${ }^{1-4}$. The sequential action of three enzymes, an ATP-dependent ubiquitin activating enzyme (E1), a ubiquitin-conjugating enzyme (E2), and a ubiquitin-ligase (E3), work to covalently attach the C-terminal glycine (G76) of ubiquitin (Ub) to target proteins, usually through formation of an isopeptide bond to a lysine side chain. Despite lacking a Ub system of their own, many pathogenic bacteria have evolved enzymes or substrate adaptors with the ability to interact with the Ub-signaling system of their eukaryotic hosts, allowing them to take control of host processes and modulate them for their benefit ${ }^{5,6}$. Usually injected into their host cytoplasm through specialized secretion systems, various bacterial effectors have been found to use an array of strategies to hijack or exploit Ub-signaling pathways. Numerous effectors have been found to function as E3 ligases that utilize the host ubiquitination machinery to target host proteins for $\mathrm{Ub}$ modification ${ }^{7}$. Other effectors work as deubiquitinases, proteases that cleave the isopeptide bonds that link $\mathrm{Ub}$ to target proteins and reverse $\mathrm{Ub}$ signals ${ }^{8,9}$. Some effectors even chemically attack and disable specific components of the eukaryotic ubiquitinating machinery directly ${ }^{10-12}$, including covalent alteration of Ub itself ${ }^{13}$. However, in recent years, our understanding of this post-translational modification has been redefined by the discovery of bacterial enzymes that catalyze Ub transfer using strategies that bypass the canonical E1-E2-E3 pathway. This was first demonstrated for the SidE family of Legionella effectors that catalyze $\mathrm{NAD}^{+}$-assisted phospho-ribosyl linked ubiquitination of certain host targets ${ }^{14-17}$.

Legionella pneumophila possesses a large arsenal of effectors with over 300 examples of proteins injected into the host via its Dot/Icm Type IV secretion system. These effectors are critical in allowing L. pneumophila to form a replicative niche within the host cell where it can survive and avoid host defense mechanisms ${ }^{18-20}$. The newly discovered L. pneumophila effector MavC serves as another fascinating example of an enzyme that targets host Ub-signaling pathways, but works in a manner that is distinctly different from the eukaryotic Ub-transfer machinery ${ }^{21,22}$ (Supplementary Fig. 1a-c). Valleau et al. ${ }^{21}$ first reported the structure of apo-MavC and described its function as a Ub-specific deamidase that catalyzes the conversion of Ub to its Glu40 variant. Together with structural analysis of the effector, the authors concluded that MavC is a structural and functional homolog of known bacterial deamidases such as Cif and $\mathrm{CHBP}^{13,23,24}$ that target the conserved Gln40 of NEDD8 and Ub. The deamidase structural core in these effectors is conserved in MavC, but MavC also features a unique "insertion" domain that is required for interaction with the E2 Ube2N (also known as Ubc13). These observations led the authors to propose the thioester-linked Ube $2 \mathrm{~N}-\mathrm{Ub}$ conjugate as the deamidation substrate to disrupt Ube2N-dependent synthesis of K63-linked Ub chains critical for the innate immune response. However, no clear demonstration of activity on this specific substrate was provided.

Subsequently, Gan et al. ${ }^{22}$ co-transfected mammalian HEK293 cells with MavC and a Ub variant that lacks its C-terminal glycine residues and hence cannot be activated or transferred via the canonical eukaryotic E1-E2-E3 pathway. In this context, MavC was observed to modify Ube2N with the $\mathrm{Ub}$ variant via a transglutamination reaction that proceeds via an obligate thioester enzyme intermediate between $\mathrm{Q} 40^{\mathrm{Ub}}$ and $\mathrm{C} 74^{\mathrm{MavC}}$. The result is the creation of an isopeptide linkage between the $\gamma$ carbonyl group of $\mathrm{Gln} 40^{\mathrm{Ub}}$ and $\varepsilon$-amino group of Lys $92^{\mathrm{Ube}} 2 \mathrm{~N}$ (Fig. 1a). Ubiquitination of Lys $92^{\text {Ube } 2 N}$, located adjacent to the $\mathrm{E} 2$ active site, effectively inhibits Ube2N activity and attenuates downstream host NF- $\mathrm{kB}$ activation ${ }^{25}$. Furthermore, the activity a
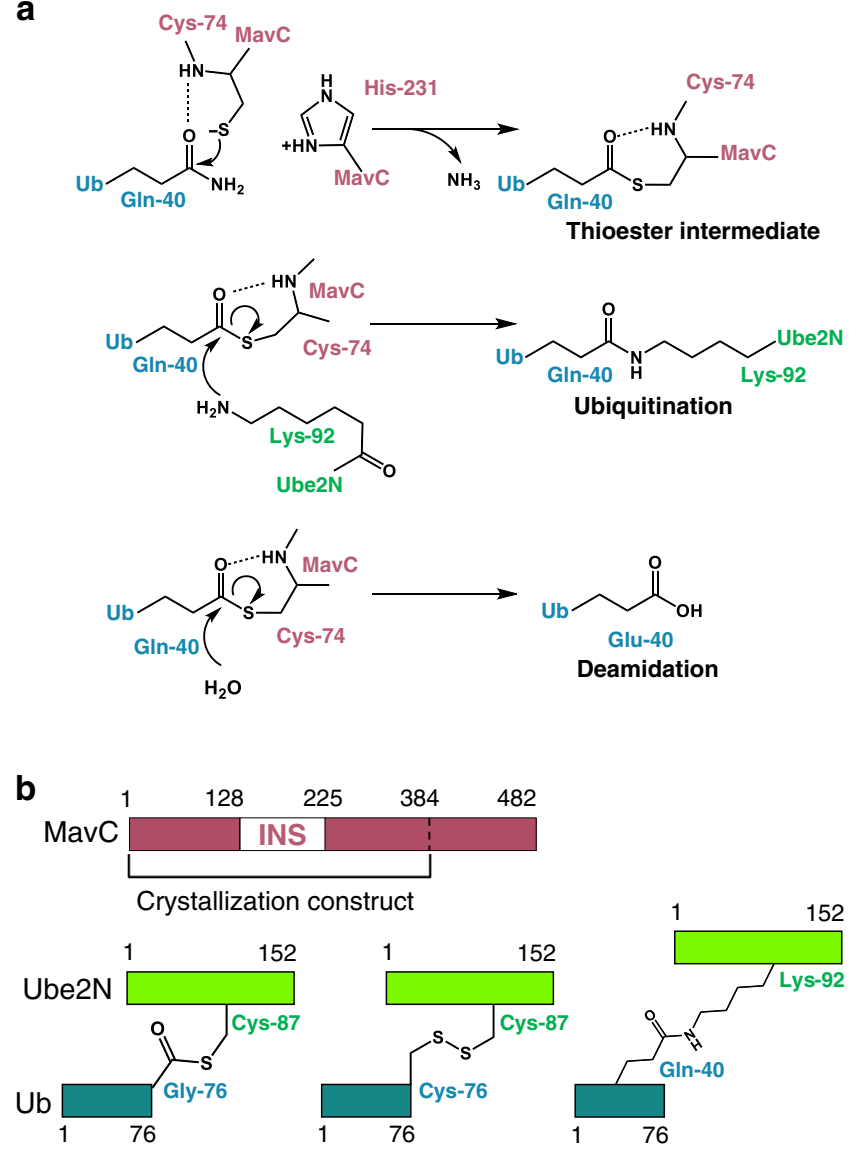

Fig. 1 Proposed mechanisms of MavC-catalyzed reactions and constructs used for structural studies. a Proposed mechanism of ubiquitination resulting from transglutamination reaction catalyzed by MavC. Thioester formed in Step 1 may undergo either attack by the amino group of Ube2N Lys92 (leading to formation of Ub-Ube2N) or hydrolysis (formation of deamidated $\mathrm{Ub}$ ). Key residues from MavC in burgundy, Ube2N in light green, and Ub in teal are represented. b Diagram depicting protein constructs used for crystallization studies and NMR experiments and location of the MavC insertion domain (residues 128-225), with diagrams of Ube2N-Ub, Ube2N-SS-Ub (MavC substrates), and Ub-Ube2N (MavC product) provided for comparison.

of MavC is antagonized by MvcA, a protein of $50 \%$ identity with MavC that functions to remove $\mathrm{Ub}$ from $\mathrm{Ub}-\mathrm{Ube} 2 \mathrm{~N}$ in later phases of infection ${ }^{26}$, which points to the importance of temporal regulation of the activity of this E2 enzyme during $L$. pneumophila infection. Thus, MavC catalyzes what seems to be the only known example of a Ub transfer reaction that does not require a nucleotide cofactor to activate $\mathrm{Ub}$ prior to substrate modification $^{22}$.

A key assumption of the previous work was that MavC recognizes and joins free $\mathrm{Ub}$ and Ube $2 \mathrm{~N}$ together ${ }^{22}$. However, in cells it has been predicted that E2s exist predominantly as the activated E2-Ub conjugate poised to transfer Ub to substrates ${ }^{27}$. Moreover, while MavC-catalyzed Ub deamidation occurs fairly slowly compared to the transglutaminase-mediated E2 ubiquitination activity ${ }^{22}$, it remains unclear how the enzyme prioritizes one activity over the other given their mutual exclusivity. Here, we demonstrate that MavC actually targets the Ube $2 \mathrm{~N}-\mathrm{Ub}$ conjugate to catalyze an intramolecular transglutamination reaction. We present a series of crystal structures of MavC in complex with a disulfide-linked substrate that mimics the charged Ube $2 \mathrm{~N}-\mathrm{Ub}$ conjugate and with the transglutaminase crosslinked product 
(Fig. 1b). These structures reveal how interaction with MavC leads to remodeling of regions surrounding the Ube2N active site to promote the intramolecular transglutaminase reaction and inhibit Ube2N-Ub function. The progression of structures reveals key features in MavC that provide a basis for understanding its transglutaminase mechanism, substrate specificity, and how conformational dynamics favor a specific reaction outcome.

\section{Results}

MavC efficiently transglutaminates a Ube2N-Ub mimic. Previous work on MavC implicated free Ube2N and/or Ub as substrates $^{21,22}$. To determine how MavC engages substrates we used biolayer interferometry (BLI) to measure binding affinities and NMR to map MavC/substrate interactions. The combined results show that free Ub binds only weakly to MavC and binding constants could not be determined with high confidence (Fig. 2a). Though an approximate $K_{\mathrm{d}}$ of $\sim 170 \mu \mathrm{M}$ for binding of free Ub to MavC was deduced from BLI measurements, reaction assays and NMR experiments suggest the affinity is much weaker: reaction assays monitoring Ub deamidation as a function of Ub concentration failed to saturate activity even at $375 \mu \mathrm{M} \mathrm{Ub}$, suggesting that the $K_{\mathrm{M}}$ for free $\mathrm{Ub}$ in this reaction is in excess of 200 $\mu \mathrm{M}$ (Fig. 2b, Supplementary Fig. 2a, b). NMR titration of ${ }^{15} \mathrm{~N}-\mathrm{Ub}$ using a catalytic Cys-to-Ala mutant of MavC (C74A-MavC $\left.{ }_{1-384}\right)$ revealed almost no chemical shift perturbations (CSPs) in the Ub spectrum at equimolar $(150 \mu \mathrm{M})$ concentrations. Further addition of $150 \mu \mathrm{M}$ Ube2N to the NMR sample did not appreciably enhance the weak interaction between MavC and Ub (Fig. 2c). We note that a previous study conducting similar NMR titration experiments, but using catalytically active MavC, reported significant CSPs in Ub upon addition of $\mathrm{MavC}^{21}$. However, we found those results consistent with the MavC-catalyzed conversion of $\mathrm{Ub}_{\mathrm{WT}}$ to the deamidated product, $\mathrm{Ub}_{\mathrm{Q} 40 \mathrm{E}}$, and not with direct protein-protein interactions (Supplementary Fig. 2c, d).

In marked contrast to Ub, Ube $2 \mathrm{~N}$ binds MavC with much higher affinity. Indeed, previous work identified a MavC/Ube2N complex in cell extracts ${ }^{21}$. Our BLI measurements yielded a $K_{\mathrm{d}}$ of $\sim 2.5 \mu \mathrm{M}$ for Ube2N (Fig. 2a). In NMR titration experiments, numerous CSPs are observed upon addition of C74A-MavC $\mathrm{C}_{1-384}$ to ${ }^{2} \mathrm{H},{ }^{15} \mathrm{~N}$-labeled Ube2N (Supplementary Fig. 2e). The observed CSPs define a MavC recognition surface on Ube2N formed by residues in Helix1, Loop4, and Loop7 (Supplementary Fig. 2f). This is the same Ube2N surface shown to interact with numerous eukaryotic E3 ligases ${ }^{28}$. An analogous NMR titration using purified MavC insertion domain $\left(\mathrm{MavC}_{128-225}\right)$ yielded Ube2N spectral changes that are remarkably similar to those observed with C74A-MavC ${ }_{1-384}$ despite the large molecular weight differences (Supplementary Fig. 2g). These observations suggest that the MavC insertion domain is primarily responsible for binding Ube2N, a conclusion supported by similar $K_{\mathrm{d}}$ values obtained from BLI measurements of Ube2N binding to MavC or the MavC insertion domain (Fig. 2a, Supplementary Fig. 1e).

Despite the relatively high affinity for Ube2N, the weak interaction between MavC and $\mathrm{Ub}\left(K_{\mathrm{M}}>200 \mu \mathrm{M}\right)$ indicates an intermolecular transglutamination reaction between free Ub and Ube $2 \mathrm{~N}$ would be unlikely to occur under cellular conditions. As most cellular E2s are predicted to have Ub tethered to its activesite Cys via a thioester linkage ${ }^{27,29}$, we set out to assess whether Ube2N-Ub is the relevant substrate for MavC. Our approach utilized a stable Ube $2 \mathrm{~N}-\mathrm{Ub}$ mimic $^{30}$ in which the $\mathrm{G} 76 \mathrm{C}^{\mathrm{Ub}}$ mutant is disulfide linked to the active site Cys 87 Ube2N (Fig. 1b). This mimic provided greater control of reaction components and minimized experimental complications that would arise from hydrolysis of purified wild-type Ube2N-Ub during MavC reaction assays and during NMR experiments. (Hereafter, this substrate surrogate is referred to as Ube2N-SS-Ub or the disulfide conjugate.)

BLI and NMR experiments show MavC readily binds Ube2NSS-Ub. BLI-binding titrations yield a $K_{\mathrm{d}}$ of $\sim 2.4 \mu \mathrm{M}$ for Ube $2 \mathrm{~N}$ $\mathrm{SS}-\mathrm{Ub}$, nearly the same affinity as observed for free Ube2N (Fig. 2a), consistent with binding dominated by interactions with Ube2N. NMR experiments were performed using a disulfide conjugate in which only the Ub subunit was isotopically labeled (Ube2N-SS- ${ }^{2} \mathrm{H},{ }^{15} \mathrm{~N}-\mathrm{Ub}$ ). In the conjugate, not all Ub resonances are observed or of equal intensity. This is due to the Ub subunit alternating between open states, where Ub makes few contacts with the E2, and closed states, where the Ub subunit is in close contact with the E2 (ref. ${ }^{31}$ ). This equilibrium results in exchange broadening of resonances whose environments differ in the open and closed states. Ub resonances that remain and largely overlap with those of free Ub can be assigned by inspection (Supplementary Fig. 2h, i).

In marked contrast to the addition of $\mathrm{MavC}$ to free $\mathrm{Ub}$ (Fig. 2c), large perturbations in the spectrum of the Ub subunit of ${ }^{2} \mathrm{H},{ }^{15} \mathrm{~N}$-Ub-SS-Ube2N are now observed upon formation of an ${ }^{2} \mathrm{H},{ }^{15} \mathrm{~N}$-Ub-SS-Ube2N/MavC complex (Fig. 2d). An overall loss in peak intensity is observed consistent with the large increase in molecular weight $(\sim 65 \mathrm{kDa})$ upon complex formation. In addition, a number of $\mathrm{Ub}$ resonances disappear. Again, this behavior can be attributed to resonance exchange broadening where a subset of Ub residues exchange between contacts with MavC, Ube2N, and/or solvent. Thus, in solution, the Ub subunit is not rigidly fixed to the enzyme active. However, the high local concentration of $\mathrm{Ub}$ provided by MavC binding of the Ube2N-Ub conjugate significantly increases observed contacts.

In targeting Ube $2 \mathrm{~N}-\mathrm{Ub}$ for modification, MavC could catalyze deamidation of the Ub subunit, an intramolecular transglutaminase reaction between the $\mathrm{E} 2$ and $\mathrm{Ub}$ subunits, or some combination of the two. In all scenarios, tethering the Cterminus of $\mathrm{Ub}$ to the $\mathrm{E} 2$ active site must not hinder the ability of MavC to form an obligate thioester intermediate with the Gln40 side chain of the Ub subunit. Furthermore, to catalyze transglutamination, MavC must be able to orient Lys 92 Ube $2 \mathrm{~N}$ and $\mathrm{Gln} 40^{\mathrm{Ub}}$ of the E2-Ub conjugate in proximity to form an isopeptide bond. To investigate these possibilities, we conducted assays using $25 \mu \mathrm{M}$ Ube $2 \mathrm{~N}-\mathrm{SS}-\mathrm{Ub}$ conjugate as substrate and enzymatic amounts of MavC $(5 \mathrm{nM})$. Under these conditions, MavC exhibits robust transglutaminase cross-linking activity, while deamidation of $\mathrm{Ub}$ of the disulfide substrate was not detected (Fig. 2e, Supplementary Fig. 2k). In sharp contrast, reactions using free $\mathrm{Ub}$ and $\mathrm{Ube} 2 \mathrm{~N}$ at the same subunit concentrations as the disulfide conjugate $(25 \mu \mathrm{M})$ produced no detectable transglutaminase product (Fig. 2e). These results strongly argue that MavC targets the Ube $2 \mathrm{~N}-\mathrm{Ub}$ conjugate to catalyze an intramolecular transglutaminase reaction resulting in the formation of an isopeptide bond between $\mathrm{Ube} 2 \mathrm{~N}$ and $\mathrm{Ub}$ These results are also in line with observations of Gan et al. ${ }^{22}$ which show that a mutant of Ub lacking the last two glycines is modified to a significantly lower extent than wild-type Ub.

Structural basis of transglutaminase-mediated ubiquitination. MavC-catalyzed transglutamination proceeds via an obligate thioester-linked intermediate to form an isopeptide bond between $\mathrm{Gln} 40^{\mathrm{Ub}}$ and Lys92 $\mathrm{Ube} 2 \mathrm{~N}$ (Fig. 1a). To gain structural insights into the mechanism underlying this noncanonical ubiquitination, we sought to crystallize MavC with both substrate and product. For crystallization trials, we used a truncated MavC construct, $\mathrm{MavC}_{1-384}$ (Fig. 1b), that otherwise retains full enzymatic activity and Ube2N binding (Supplementary Fig. 1d, e). In addition, the $\mathrm{MavC}$ active site residue, Cys74, was mutated to $\mathrm{Ala}(\mathrm{C} 74 \mathrm{~A})$ to 
a

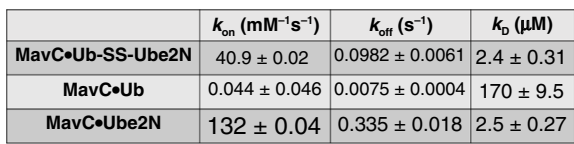

C

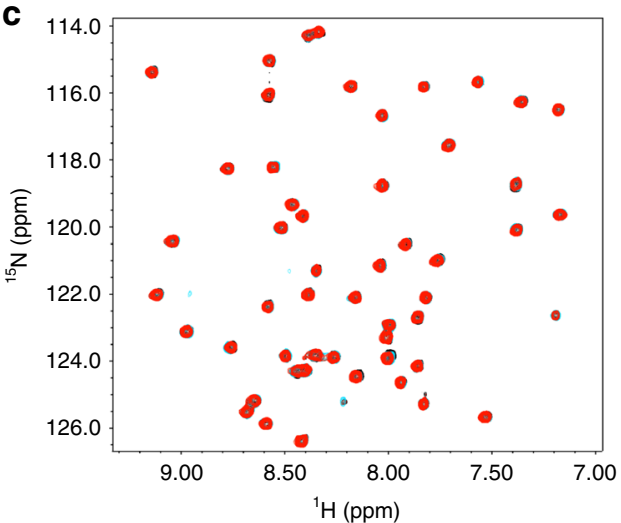

e

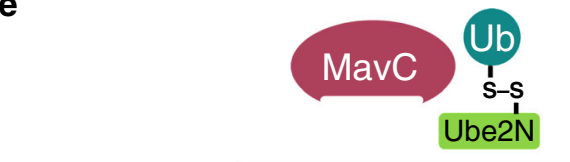

b

\begin{tabular}{|c|c|c|}
\hline & Deamidation & Ubiquitination \\
\hline $\boldsymbol{k}_{\mathrm{M}}(\boldsymbol{\mu M})$ & $688.3 \pm 235.5$ & $10.4 \pm 4.3$ \\
\hline $\boldsymbol{k}_{\text {cat }}\left(\mathbf{s}^{-1}\right)$ & $0.12 \pm 0.03$ & $0.024 \pm 0.004$ \\
\hline
\end{tabular}

d
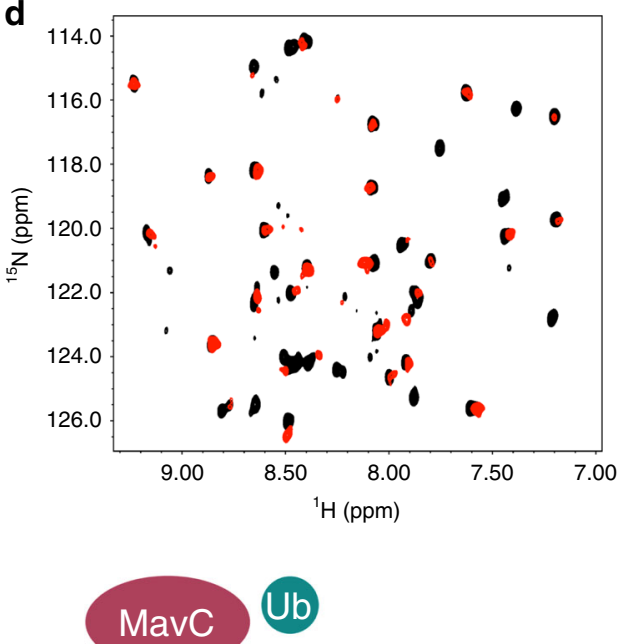

Ube2N
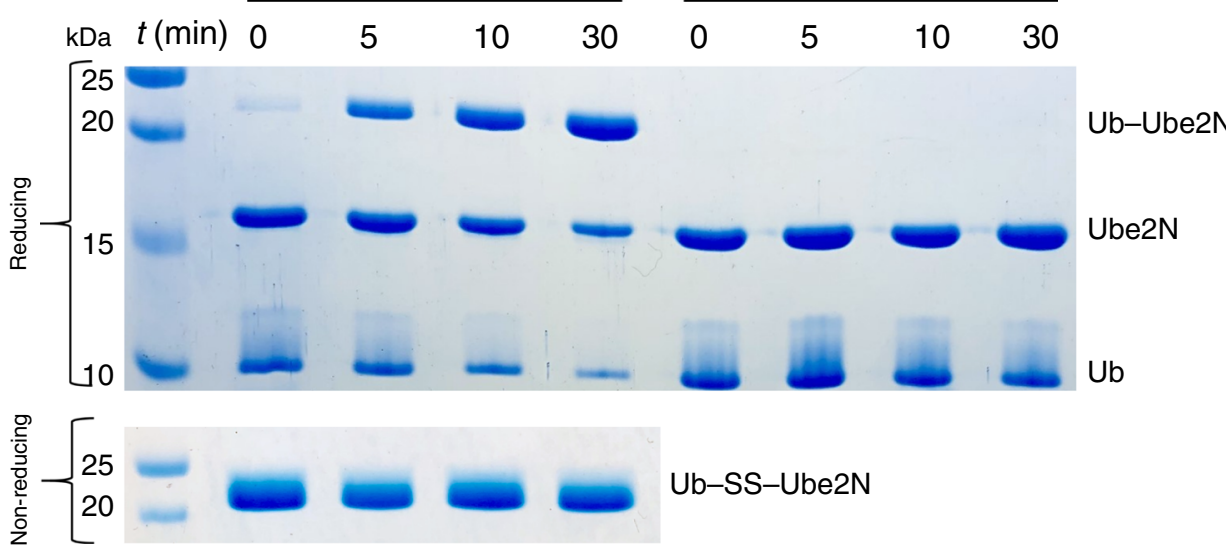

Fig. 2 Ube2N-SS-Ub is a significantly better substrate for MavC than free Ub/Ube2N. a Biolayer interferometry table showing the measured on and off rates and the dissociation constants of MavC (C74A) with Ube2N-SS-Ub, Ub, and Ube2N. b Michaelis-Menten parameters of ubiquitination (with respect to Ube2N) and deamidation (with respect to Ub) reactions. c Free Ub binds weakly to MavC. ${ }^{1} \mathrm{H},{ }^{15} \mathrm{~N}-\mathrm{HSQC}$ NMR spectral overlays of $150 \mu \mathrm{M}{ }^{15} \mathrm{~N}-\mathrm{Ub}$ alone

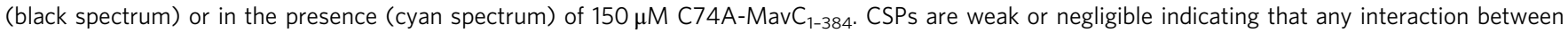
MavC and free $\mathrm{Ub}$ is very weak. Addition of $150 \mu \mathrm{M}$ Ube2N to the sample (red spectrum) has marginal effects on the Ub spectrum, and therefore, does not

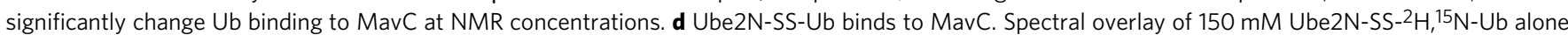

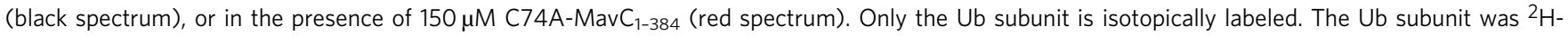
labeled to allow for NMR observation in high molecular weight complexes. Select Ub subunit resonances are observed to shift or disappear indicating that the Ub subunit interacts with MavC. e A time course comparison of the ubiquitinating activity of MavC on Ube2N-SS-Ub versus free Ube2N and Ub as the substrates. Loading controls for Ube2N-SS-Ub for each time point under non-reducing conditions are also shown. Reactions were subjected to SDS-PAGE and visualized with Coomassie Blue. A control reaction at time 0 is included. Accompanying each time course is a cartoon representation of the reaction components.

prevent any modification of Ub or Ube $2 \mathrm{~N}$ during crystallization. The MavC/substrate complex was generated using the substrate mimic Ube2N-SS-Ub used in NMR studies and in biochemical assays (see Fig. 2). The substrate complex crystallized in three different space groups (Table S1), C222, R3, and $\mathrm{P}_{5}$. The product complex was generated using $\mathrm{Ub}-\mathrm{Ube} 2 \mathrm{~N}$ product isolated from a MavC-catalyzed reaction mixture where free Ube $2 \mathrm{~N}$ and $\mathrm{Ub}$ were linked via a $\gamma$-glutamyl- $\varepsilon$-Lys isopeptide link.

The structure of $\mathrm{MavC}_{1-384}$ in all complexes (Fig. 3) is topologically identical to that in the previously determined structure of apo- $\mathrm{MavC}_{1-384}$ (PDB id 5TSC ${ }^{21}$ ). Architecturally, $\mathrm{MavC}_{1-384}$ is composed of three distinct lobes: a core globular domain (CG) flanked by an insertion domain (residues 128-225) on one end and an a-helical extension (HE: residues 33-66, 356-384) on its opposite end. The overall structure of $\mathrm{MavC}_{1-384}$ can be described as a C-shaped crescent with a crevice where the catalytic center is located (Fig. 3). Looking down into the concave face of the crescent, the MavC catalytic triad (residues Cys74 $(\mathrm{C} 74 \mathrm{~A})$, His231, and Gln252) is located near the center of the C-shaped structure (Fig. 3). In all structures, the Ube $2 \mathrm{~N}$ and $\mathrm{Ub}$ subunit bind in an extended conformation, with Ube $2 \mathrm{~N}$ on one side of the active site and Ub on the other. The Ub subunit binds between the insertion domain and the $\mathrm{HE}$, positioning the $\mathrm{Gln} 40^{\mathrm{Ub}}$ side chain within the MavC catalytic cleft. The structures 

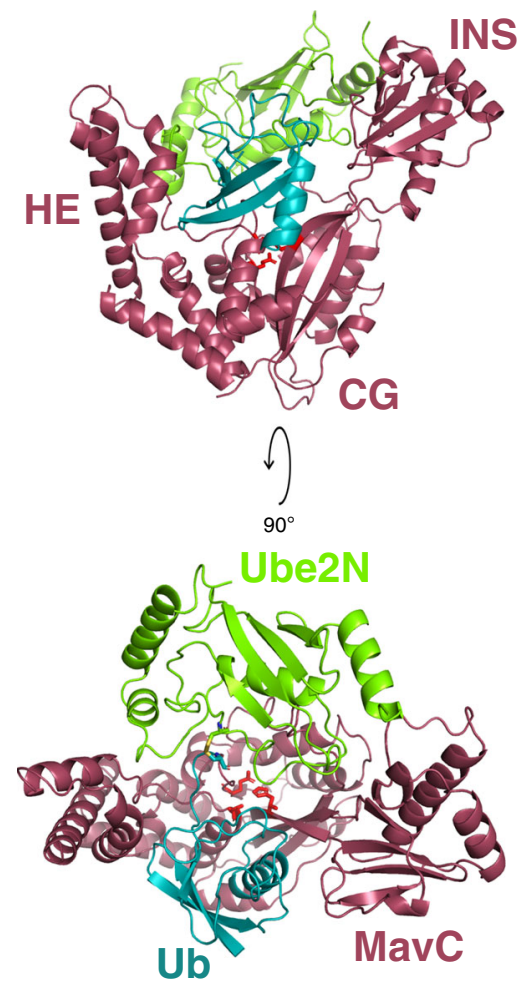

Fig. 3 Overall structure of Ube2N-SS-Ub bound to MavC. Cartoon representation of the crystal structure of MavC $\bullet$ Ube2N-SS-Ub complex $\left(\mathrm{P}_{5}\right)$, with MavC depicted in burgundy, Ube2N in green, and $\mathrm{Ub}$ in teal. Key domains (CG, core globular domain; $\mathrm{HE}$, helical extension; INS,

insertion domain) are labeled. Rotated view depicting a top-down view of the active site is also shown. MavC active site residues $\mathrm{C} 74, \mathrm{H} 231$, and Q252 are depicted as red sticks.

are consistent with our solution NMR binding experiments. The surface of the Ube $2 \mathrm{~N}$ subunit formed by Helix1, Loop4, and Loop7 makes extensive contacts with the MavC insertion domain (Fig. 3, Supplementary Fig. 2f). Observable Ub resonances not significantly affected by complex formation are very similar to those of free Ub (Supplementary Fig. 2h, i). These resonances can be assigned by inspection and are primarily in located in regions of the Ub subunit that do not make contact with MavC (Supplementary Fig. 2j).

Though the Ub subunit does not bind tightly to MavC in solution, the structures reveal a number of potential contacts that we found important for activity. Residues in the MavC HE region (MavC Leu36, Asn39, Glu40, Ile43, and Glu66) interact with the $\mathrm{N}$-terminal $\beta$-hairpin turn of $\mathrm{Ub}$ as well as the nearby $\mathrm{C}$-terminal Ub tail (Ub Leu8, Thr9, Arg72, and Arg74) (Fig. 4a, b). Around the MavC catalytic triad, numerous contacts are observed, including a rare contact with the di-Pro motif of $\mathrm{Ub}$ (Pro37-Pro38) using a $\mathrm{CH}-\mathrm{O}$ hydrogen bond between the carbonyl group of Ala $229^{\mathrm{MavC}}$ and $\mathrm{H} \delta$ atoms of the Pro38 $\mathrm{Ub}$ (ref. ${ }^{32}$ ) (Fig. 4b). Numerous hydrophilic contacts include Asn $72^{\mathrm{MavC}}$ positioned to $\mathrm{H}$-bond with the backbone carbonyl of Leu73 $\mathrm{Ub}, \operatorname{Arg} 121^{\mathrm{MavC}}$ in H-bonding distance to the carbonyl of $\mathrm{G} 35^{\mathrm{Ub}}$, and the side chains of $\mathrm{R} 126^{\mathrm{MavC}}$ and Thr230 MavC poised to interact with the Asp39 $\mathrm{Ub}$ carboxylate. Mutation of these MavC residues leads to a reduction or complete loss of the ability of MavC to catalyze either transglutamination (using the disulfide conjugate as a substrate) or Ub deamidation (Fig. 4c, d). Mutation of Arg72 $\mathrm{Ub}$, one residue that distinguishes Ub from the structurally related NEDD8, to alanine severely impairs the ability of MavC to recognize this mutant substrate (Supplementary Fig. 3a).

Modeling a Cys side chain in a preferred rotamer orientation in place of MavC Ala74 reveals the $\gamma$-S atom poised for nucleophilic attack at $\mathrm{Gln} 40^{\mathrm{Ub}}$, approaching within $3.0 \AA$ of the carboxamide group (Fig. 4e). Gln $40^{\mathrm{Ub}}$ is held in the active site with its side chain $\mathrm{C}=\mathrm{O}$ group pointing toward the backbone amide groups of the catalytic Cys74 $4^{\mathrm{MavC}}$ and Trp255 $5^{\mathrm{MavC}}$ (H-bonding distance of 2.9 and $3.0 \AA$, respectively) whereas the $\mathrm{NH}_{2}$ group is in $\mathrm{H}$ bonding contact with the backbone carbonyl group of Thr230 MavC $(2.8 \AA)$ and the imidazole side chain of His231 (Fig. 4e). The backbone amides would be important for stabilization of the oxyanion transition state and the His231 interaction is likely for proton donation to the leaving ammonia during the thioester step (Fig. 1a). The indole side chain of $\operatorname{Trp} 255^{\mathrm{MavC}}$ is stacked against the Gln40-Gln41 peptide unit of $\mathrm{Ub}$ (Fig. 4e), an arrangement that permits the backbone carbonyl of $\mathrm{Gln} 40^{\mathrm{Ub}}$ to come within H-bonding distance from the hydroxyl group of Ser73 $3^{\text {MavC }}$. Mutation of Trp255 and Ser73 of MavC to alanine results in a significant loss of Ub deamidation and transglutamination activity of the enzyme (Fig. 4c, d). Combined, these interactions appear to fix the $\mathrm{Gln} 40^{\mathrm{Ub}}$ side chain in a reactive arrangement for attack by the nucleophilic Cys to facilitate formation of the thioester intermediate. In this arrangement the $\mathrm{NH}_{2}$ group points toward a solvent-filled area, which would allow the ammonia produced during catalysis to diffuse away from the active site.

In both substrate and product complexes, there are three regions of MavC that interact with Ube2N. Region 1, the MavC insertion domain, makes an overwhelming contribution to Ube2N binding compared to other parts of MavC. Around 500 $\AA$ (ref. ${ }^{2}$ ) of the surface area is buried at the interface between $\mathrm{MavC}_{\mathrm{INS}}$ and Ube2N alone. Accordingly, its deletion results in a dramatic loss of Ube $2 \mathrm{~N}$ binding and no detectable ubiquitination activity (Fig. 5d) ${ }^{21}$. The MavC insertion domain can be expressed and purified on its own, and a crystal structure of the insertion domain shows that it preserves an identical fold to that in the full MavC protein (Supplementary Fig. 4a). BLI and glutathione-stransferase (GST) pulldown experiments show that the isolated insertion domain is able to bind to Ube $2 \mathrm{~N}$ independently and with an affinity comparable to full-length MavC (Supplementary Fig. $4 b, c)$. The second Ube $2 \mathrm{~N}$-interacting region of MavC corresponds to residues in the $\mathrm{CG}$ domain, particularly Met $317^{\mathrm{MavC}}$, that interacts with Loop4, the $3{ }_{10}$-helix containing Lys $92^{\text {Ube } 2 \mathrm{~N}}$, and $a$ Helix 2 of Ube $2 \mathrm{~N}$ (Fig. 5b). The third region involves contacts between the MavC $\mathrm{HE}$ domain and Ube2N aHelix3 (Fig. 5c), an interface largely supported by a network of polar contacts between acidic and basic residues from both MavC and Ube2N. The contacts in this region appear to play a key role as the substrate transitions through the catalytic process. For example, interactions involving Arg63 $3^{\mathrm{MavC}}$ and Lys64 ${ }^{\mathrm{MavC}}$ with the aHelix 3 of Ube2N are observed only in the $\mathrm{P}_{5}$ substrate complex structure, which is closer to the product complex (also in $\mathrm{P}_{5}$ ) than the other substrate complexes. Consistent with these observations, mutation of Arg63 MavC Lys64 ${ }^{\mathrm{MavC}}$, and Glu66 ${ }^{\mathrm{MavC}}$

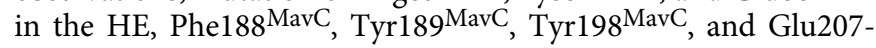
MavC in the insertion domain, and Met317MavC in the CG severely impair MavC catalytic activity (Fig. 5d). Tyr47 MavC was also chosen as a site for mutation in these mutants because it contributes to hydrophobic interactions at the HE interface. Based on results from the biochemical experiments, we examined the effects of a Tyr47/Tyr198/Glu207MavC to alanine triple mutant (the YYE mutant) on MavC-induced Ube2N ubiquitination in cells infected by L. pneumophila. We also created mutants by additionally changing either Met 317 or Trp255 to alanine in 
a

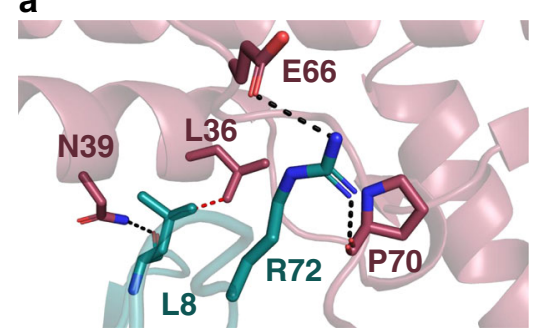

c

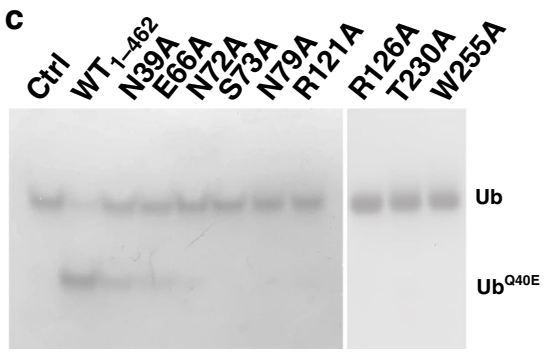

e

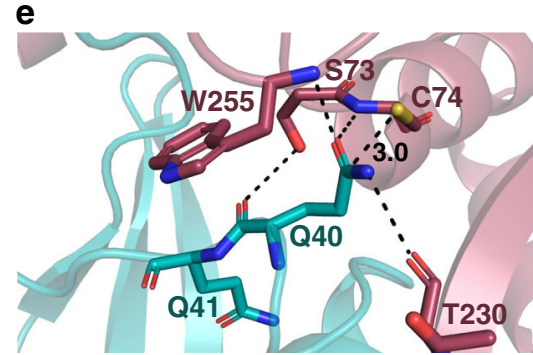

b
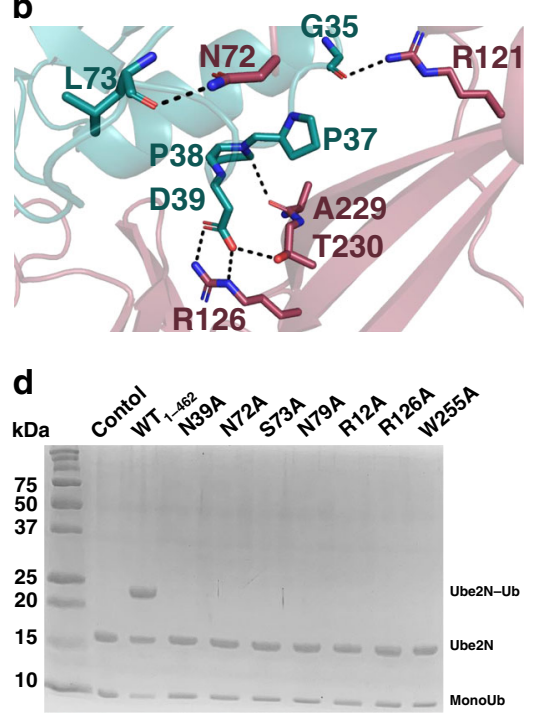

Fig. 4 Binding interface of MavC with Ub. a, $\mathbf{b}$ Views of the MavC •Ub-binding interface showing interactions between residues of MavC (burgundy) and Ub (teal). Hydrogen-bonding interactions are shown in black dashed line and hydrophobic contacts in red dashed line. c Native-PAGE gel comparing Ub deamidation activities of Ub-binding mutants of MavC. Reactions were visualized with Coomassie Blue. A control reaction without MavC is included. d Comparison of the ubiquitinating activity of the Ub-binding mutants of MavC using Ube2N-SS-Ub as the substrate. Reactions were subjected to SDSPAGE and visualized with Coomassie Blue. A control reaction without MavC is included. e Architecture of the active site in the structure of MavC substrate complex (P65), with Ala74 of MavC modeled as a Cys. Interactions holding Gln40 ${ }^{\mathrm{Ub}}$ in position are described.

the YYE mutant (YYE/M317A, YYE/W255A). Unlike the single point mutants or YYE, which show appreciable activity under longer reaction times in the transglutaminase assay, these quadruple mutants were much more defective (Supplementary Fig. 3b, c). All of these mutants were translocated into infected cells at levels comparable to that of the wild-type complementation. We then examined the levels of Ube2 $\mathrm{N}$ ubiquitination in infected cells. We also examined the ability of MavC and the mutants to attenuate NF- $\kappa \mathrm{B}$ activation under conditions of TRAF6 overexpression ${ }^{22}$. Whereas the activity of the YYE mutant has severely impaired the ability to modify Ube $2 \mathrm{~N}$ and displays defects in attenuating NF- $\mathrm{BB}$ activation, the quadruple mutants were further impaired to levels comparable to the catalytically inactive $\mathrm{C} 74 \mathrm{~A}$ mutant, in line with the biochemical activity assay results (Fig. 5e, f, Supplementary Fig. 3c). Collectively these results indicate that the transglutaminase activity of MavC targeting the Ube $2 \mathrm{~N}-\mathrm{Ub}$ conjugate is essential for its biological role in attenuating NF- $\mathrm{KB}$ response in host cells.

MavC remodels the Ube2N active site to promote crosslinking. Remarkably, comparison of substrate and product MavC complexes reveal a progression in the conformation of loops surrounding the Ube $2 \mathrm{~N}$ active site. In one structure containing Ube2N-SS-Ub (Fig. 6a), the E2 conformation is very similar to 49 other structures of Ube2N available in the Protein Data Bank
(Supplementary Fig. 4d). The Ube2N active site loop formed by residues $116-123$ is the most variable region among Ube2N structures, and in the MavC complex it adopts an altered conformation relative to an average Ube2N structure (Supplementary Fig. 4d). Lys $92^{\text {Ube2N }}$, the residue that will form an isopeptide

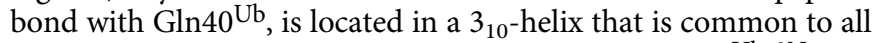
other Ube2N structures. Though the complete Lys92 Ube2N side chain is not resolved in this structure, its $\beta$-carbon is positioned over $16 \AA$ away from the $\gamma$-carbon of $\mathrm{Gln} 40^{\mathrm{Ub}}$. This placement is too far for reaction with a thioester intermediate and isopeptide bond formation, suggesting that a conformational change is required to bring Lys 92 Ube $2 \mathrm{~N}$ into the MavC active site. The other substrate and product MavC complexes reveal a dramatic change in the conformation of the $310^{-}$-helix that brings the Ube $2 \mathrm{~N}$ target lysine into position to attack the $\gamma$-carbon of $\mathrm{Gln} 40^{\mathrm{Ub}}$ (Fig. $6 \mathrm{a}-\mathrm{c}$ show the three states we call "early substrate", "attacking substrate," and "product", respectively, with respect to the transition of the $\left.310^{-h e l i x}\right)$. A methionine in MavC, Met317 MavC, appears to play a critical role in promoting this conformational change. In the early substrate/MavC structure (Fig. 6a), Met317MavC is positioned just below Ube2N Helix-2 and adjacent to the $33_{10^{-}}$ helix. In the attacking substrate/MavC complex, the electron density for the 310 -helix is lost suggesting this region adopts multiple conformations in the crystal. Here, Met $317^{\text {MavC }}$ has shifted $\sim 4.5 \AA$ relative to the $\mathrm{E} 2$ into a hydrophobic pocket of the E2 formed by the movement of the 310 -helix. In the product 
a

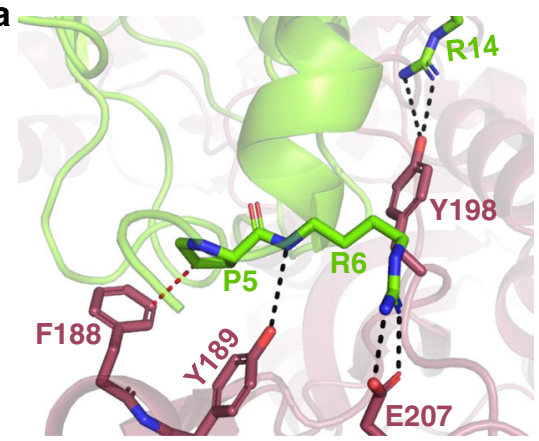

C

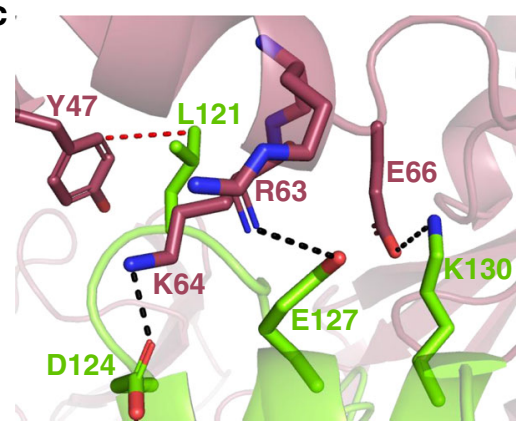

e

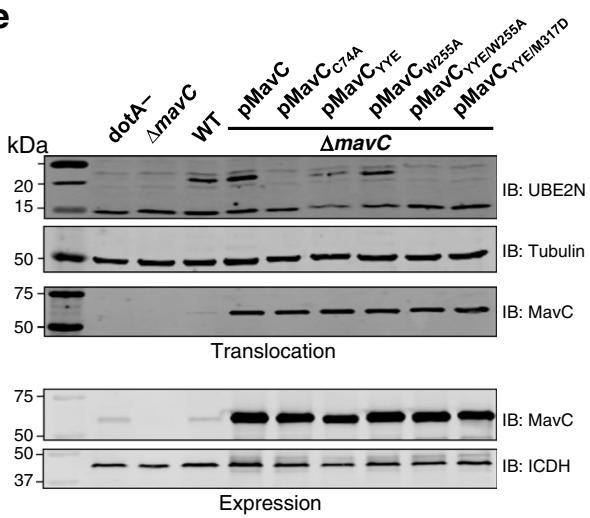

b

d
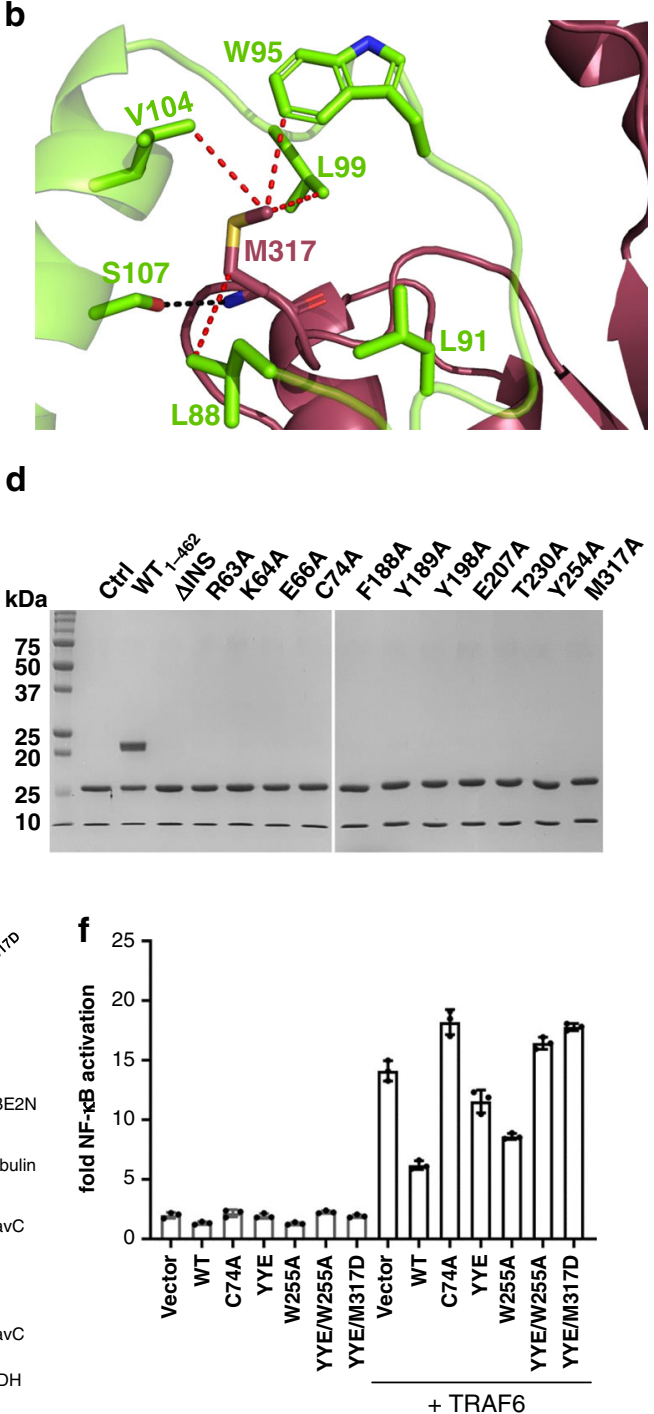

(kDa) $75-$

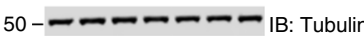

Fig. 5 Binding interface of MavC with Ube2N. a-c Detailed view of interactions in MavC regions 1, 2, and 3 with Ube2N. Key residues are labeled and represented as stick models. MavC is depicted in burgundy and Ube2N in green. Hydrogen-bonding interactions are given in black and hydrophobic interactions in red. $\mathbf{d}$ Comparison of the ubiquitinating activity of wild-type MavC versus mutant proteins using Ube2N-SS-Ub as the substrate. Reactions were subjected to SDS-PAGE and visualized with Coomassie Blue. A control reaction without MavC is included. e MavC-mediated ubiquitination of Ube2N during L.p infection. Cells infected with the indicated L. pneumophila strains were lysed with $0.2 \%$ saponin and lysate separated by SDS-PAGE, probed by immunoblotting with antibodies specific for Ube2N (upper panel) and MavC (lower panel). respectively. $\mathbf{f}$ The effects of MavC and its mutants on NFKB activation. HEK293T cells were transfected with plasmids expressing a luciferase reporter responsive to NF- $\mathrm{KB}$ and Flag-MavC or its mutant. At the same time, a plasmid expressing Renilla luciferase used as an internal control and stimulator TRAF6 were co-transfected. NF- $\mathrm{kB}$ activity was determined by dual luciferase assay. The expression of MavC and its mutants was probed in lysates of transfected cell while tubulin was detected as a loading control. Three independent experiments were done with similar results. Error bars indicate standard error of the mean (SEM).

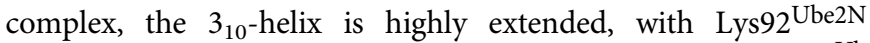
positioned in the MavC active site covalently linked to $\mathrm{Gln} 40^{\mathrm{Ub}}$ via an isopeptide bond. Notably, the methyl group of Met317 MavC occupies the position vacated by Ile $90^{\mathrm{Ube} 2 \mathrm{~N}}$ as the $3_{10}$-helix unfolds. Consistent with a role in stabilizing the extended Ube2N conformation, mutation of Met317 MavC abrogates MavC transglutaminase activity (Fig. 5d).

Additional interactions may be important for stabilizing the extended structure of Ube2N. In the product complex, the aliphatic portion of the Lys 92 side chain is held in place by van der Waals interactions with Tyr254 MavC (Fig. 6c, inset) positioning the $\varepsilon$-amino group within $4.2 \AA$ from $\mathrm{C} \beta$ of Ala74. The proximity of the backbone carbonyl of Thr $230^{\mathrm{MavC}}$ to Lys92 also indicates a potential hydrogen bond that may stabilize the Lys amino group in a productive orientation. Though the side chain of Lys 92 Ube $2 \mathrm{~N}$ is well-defined in the product complex, the region of Ube $2 \mathrm{~N}$ surrounding this residue still appears to be dynamic. This is inferred from weaker electron density and the relatively high average B-factor of $\sim 70 \AA^{2}$ for the residues from 86 to 95 , compared to $\sim 40 \AA^{2}$ for the whole complex. Thus, it appears that interactions between Ube2N-Ub and MavC lead to large conformational changes in regions surrounding the Ube $2 \mathrm{~N}$ active site, especially unfolding of the Ube2N $3_{10}$-helix, which is necessary to position Lys 92 Ube2N into the MavC active site. 


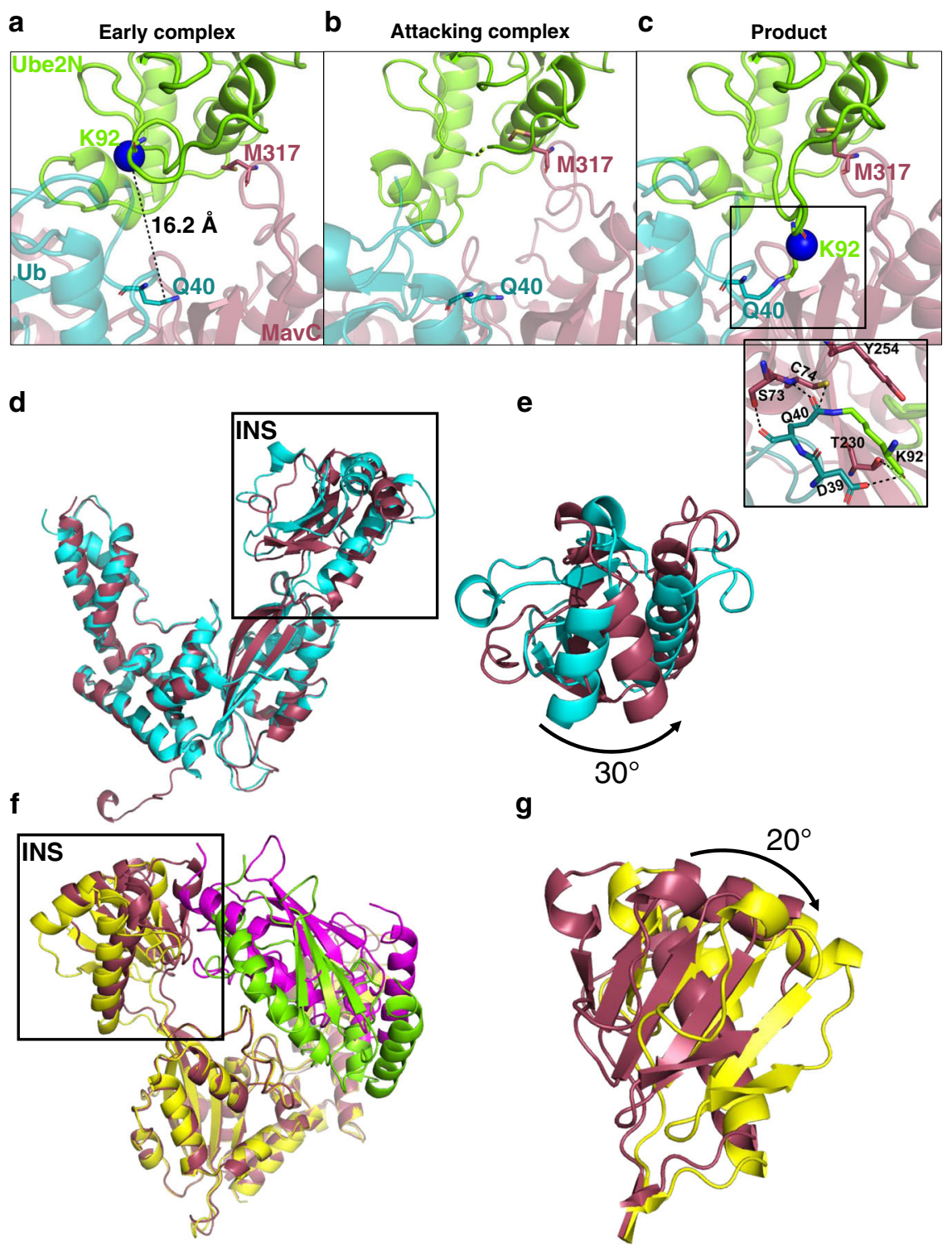

Fig. 6 Conformational dynamics of MavC and remodeling of the Ube2N active site. Superposition of Ube2N in three different structures (a-c) reveal the remodeling of Ube2N loops that occurs upon interaction with MavC. A comparison of crystal structures of MavC bound to Ube2N-SS-Ub in the $\mathrm{P} 6_{5}$ space group (a), in the R3 space group (b), and MavC bound to the product Ub-Ube2N (c) captures the progression of the $3_{10}$-helix of Ube2N from canonically structured to destabilized to unfolded. The $\beta$-carbon of the target Lys $92 \mathrm{Ube} 2 \mathrm{~N}$ is highlighted with a blue sphere. Note the changing position of $\mathrm{MavC}_{317}$ that accompanies unfolding of the Ube $2 \mathrm{~N} 3_{10}$ helix. Inset c shows interactions involving Lys $92 \mathrm{Ube} 2 \mathrm{~N}$ in the active site of MavC as observed in the productbound structure. d Structural alignment of apo-MavC (cyan) with substrate-bound MavC (R3, burgundy). Insertion domain is highlighted with a box. e Zoomed view of the insertion domain of apo and bound MavC (color coded as previously), depicting the rigid-body rotation upon Ube2N-SS-Ub binding. f Structural alignment of the two substrate-bound crystal structures of MavC (R3: MavC in burgundy, Ube2N in green. P65: MavC in yellow, Ube2N in magenta). Insertion domain is highlighted with a box. $\mathbf{g}$ Zoomed view of insertion domain in both substrate-bound crystal forms of MavC depicting the pendulum rotation required to bring Ube2N near active site.

Conformational dynamics of the MavC insertion domain. Comparison of the structure of apo-MavC (PDB id 5TSC) to the substrate complexes reveals that Ube $2 \mathrm{~N}$ could not bind to the insertion domain in apo-MavC without steric interference from the MavC HE. However, our NMR titration data show that Ube $2 \mathrm{~N}$ readily binds $\mathrm{MavC}$ in solution (Supplementary Fig. 2e, g). This suggests that flexibility of the insertion domain relative to the MavC CG and HE domains are important for function. Indeed, comparison of apo-MavC with substrate-bound complexes reveals that insertion domain undergoes a pronounced $30^{\circ}$ rigid-body rotation (Fig. 6d, e) that would enable MavC to accommodate the Ube $2 \mathrm{~N}-\mathrm{SS}-\mathrm{Ub}$ substrate. As the reaction proceeds further, the insertion domain undergoes a second rigid-body pendulum movement that helps to bring Ube $2 \mathrm{~N}$, and the side chain of Lys92 Ube2N, into the active site (Fig. $6 \mathrm{f}, \mathrm{g}$ ). Thus, MavC catalysis involves binding of Ube2N-Ub to the MavC insertion domain, motion of the insertion domain to allow additional contacts of the $\mathrm{E} 2$ and $\mathrm{Ub}$ subunits with the MavC HE and CG domains, and remodeling of the Ube2 ${ }_{3}$-helix to position Lys $92^{\text {Ube } 2 \mathrm{~N}}$ in the MavC active site for isopeptide bond formation.

MavC can target the Uev1a/Ube2N-Ub conjugate. Essential for Ube $2 \mathrm{~N}$-dependent activation of NF- $\kappa \mathrm{B}$ is the synthesis of K63linked poly-Ub chains. This requires complex formation of 
Ube2N with an $\mathrm{E} 2$ variant protein, such as Uev1a, that binds the acceptor $\mathrm{Ub}$ and directs synthesis of K63 linkages. Thus, it is likely that MavC targets Uev1a/Ube2N-Ub conjugates in order to disrupt NF- $\kappa \mathrm{B}$ activation. Uevla binds tightly to Ube $2 \mathrm{~N}^{33}$, interacting with residues in $\beta$-strands 3 and 4 of Ube $2 \mathrm{~N}$ and some of the interconnecting loops, including the extended loop that leads into the E2 active site. Our structures predict that this interaction site is solvent accessible in the MavC complex, and that MavC should also be able to target the Uev1a/Ube2N-Ub conjugate. Accordingly, we demonstrated that Uev1A can bind to Ube2N-SS-Ub as deduced from co-elution of the two proteins during size-exclusion chromatography (Supplementary Fig. 5a). This prediction is also borne out by in vitro activity data that shows increasing additions of Uevla to the reaction mixture does not inhibit the ability of MavC to catalyze the intramolecular transglutamination of Ube $2 \mathrm{~N}$ and $\mathrm{Ub}$ (Supplementary Fig. 5b). In agreement with these results, our structural analysis reveals that Uev1A binding to Ube2N-Ub is unlikely to interfere with MavC binding (Supplementary Fig. 5c).

\section{Discussion}

MavC catalysis presents a remarkable example of a ubiquitination reaction achieved through transglutaminase chemistry that does not require nucleotide-dependent activation of $\mathrm{Ub}$. The result is isopeptide crosslinking of Ub to a specific target, Ube $2 \mathrm{~N}$. Though other E2s harbor a structurally equivalent target lysine residue, selectivity for Ube $2 \mathrm{~N}$ is achieved by binding the same interface recognized by cognate eukaryotic E3 ligases (Supplementary Fig. 3b-d). Furthermore, low concentrations of MavC (nM) effectively target and inhibit both activated Ube $2 \mathrm{~N}-\mathrm{Ub}$ conjugate and the Uevla/Ube2N-Ub complex by catalyzing the synthesis of an intramolecular isopeptide bond adjacent to the E2 active site. Catalysis requires extensive remodeling of loops surrounding the E2 active site, structural changes that have not been previously observed in other Ube2N structures. Targeting a specific E2-Ub conjugate allows L. pneumophila to modulate specific host cellular processes instead of a systemic effect on the entire host Ub landscape.

With an approximate cellular concentration of $2 \mu \mathrm{M}$, Ube $2 \mathrm{~N}$ is among the most abundant E2 enzymes in cells, existing mostly as the thioester-linked Ube $2 \mathrm{~N}-\mathrm{Ub}$ conjugate 27,34 . Accordingly, MavC might have evolved to target this specific form of the E2 wherein transglutaminase catalysis would involve intramolecular crosslinking between $\mathrm{Ub}$ and $\mathrm{E} 2$ subunits of the conjugate. Notably, the binding affinity of MavC for this substrate (Fig. 2a) is within the range of its estimated cellular concentration. As a translocated effector from the phagosome, MavC is likely present at extremely low levels in the host cytosol, where such matching of substrate availability and binding affinity would ensure efficient targeting of the desired substrate. As Ube $2 \mathrm{~N}$ itself is a constitutive part of the enzymatically active heterodimeric complex with Uevla (or Uev2a), the actual in vivo substrate of MavC is most likely the heterodimeric complex, the Uevla:Ube2N-Ub complex $^{35}$. In line with this notion we find that Uevla binding does not interfere in the ubiquitination activity of MavC (Supplementary Fig. 5b). Inactivation of the Uev1a:Ube2N-Ub complex through MavC-mediated Ub crosslinking to the E2 would result in inhibition of synthesis of Lys63-linked poly-Ub chains ultimately affecting NF-kB activation. This inhibition may occur due to blocking of re-charging of Ube $2 \mathrm{~N}$ by the E1 enzyme, and also by competitive displacement of E3 binding (Fig. 7b, Supplementary Fig. 5d, e).

Although first reported to be a Ub-specific deamidase ${ }^{21}$, we find that MavC has weak affinity for free Ub, that is far too weak for a meaningful level of Ub deamidation activity under cellular conditions. This may provide an explanation for why deamidated Ub was undetectable in cells infected with wild-type $L$. pneumophila ${ }^{21,22}$. The ubiquitinating machinery of MavC is constructed from a deamidase core that is shared among previously characterized bacterial Ub/NEDD8 deamidases, to which is appended an insertion domain that serves as the E2 recruitment element. The binding affinity for Ube2N, largely contributed by the insertion domain, is relatively high, perhaps enabling MavC to compete with host E3 ligases that recruit the same E2. We propose that by targeting Ube $2 \mathrm{~N}-\mathrm{Ub}$ the enzyme leverages high-affinity interactions with the E2 subunit to effectively increase the local Ub concentration, thereby circumventing its low affinity for free Ub. Ub is seen nestled in its MavC-binding pocket in an almost identical fashion in all four structures reported here, in support of an effective increase in Ub affinity when it is presented in the format of a unit tethered to Ube2N. Additionally, having both the acyl acceptor (Lys92 ${ }^{\text {Ube2N }}$ ) and acyl donor $\left(\mathrm{Gln} 40^{\mathrm{Ub}}\right)$ units in one tethered molecule permits efficient capture of the Ub-thioester intermediate through a transamidation reaction rather than allowing a futile reaction via hydrolysis (deamidation). Even using the disulfide substrate as a means to provide $\mathrm{Ub}$ to MavC at higher affinity, we found no detectable deamidation of Ub, whereas CHBP can efficiently deamidate Ub in the same substrate (Supplementary Fig. 2k). Altogether, the results indicate that the acquisition of the insertion domain by MavC and its evolution into a Ube $2 \mathrm{~N}$-binding motif has shifted the balance in favor of the transglutamination reaction at the cost of Ub deamidation.

The structures of the substrate complexes along with that of the product-bound enzyme captured here provide striking details of the ubiquitination reaction from crystallographic snapshots indicating conformational changes along the reaction coordinate. The insertion domain appears to behave as an independent Ube2N-binding domain but when built into MavC its rotational states provide specific functionality during the transamidation reaction. We propose the following model to capture the essential features of substrate recognition and transglutaminase-mediated ubiquitination catalyzed by MavC (Fig. 7a). Initial substrate engagement by the enzyme involves recruitment of the E2 component of a conjugate through interactions primarily involving the insertion domain. This is accompanied by rotation of the insertion domain to bring the E2 into an approximate productive arrangement and allowing placement of Ub into its binding pocket, as captured in two of the structures of the substrate complexes (C222 1 and R3). A further pendulum-like rotation of the insertion domain maximizes interaction of the $\mathrm{E} 2$ with MavC by pulling the E2 closer toward the active site, as captured in the $\mathrm{P}_{5}$ substrate complex and in the product complex. In all the substrate-bound structures, the Ub subunit is ready to undergo the first step of the reaction-attack of the Cys $74^{\mathrm{MavC}}$ at $\mathrm{Gln} 40^{\mathrm{Ub}}$. The resulting thioester intermediate is protected by the distinctly hydrophobic nature of the MavC active site pocket (Tyr254 and Trp255), which faces the $3{ }_{10}$-helix of Ube $2 \mathrm{~N}$ carrying the key Lys. Unfolding of this helix brings the Lys residue into an attacking position in the active site, as captured in the product-bound complex.

The structurally related L. pneumophila effector MvcA has recently been found to use a broadly similar interface, driven by the insertion domain, to bind Ube2N. However, it catalyzes the reverse reaction of $\mathrm{MavC}$, removing $\mathrm{Ub}$ from Ube $2 \mathrm{~N}$ through hydrolysis and thereby serving to regulate MavC's activity ${ }^{26}$. Further work will be required to identify the basis of this key difference.

While MavC shares the Ub-binding site and core fold of the Cif family, our results show that it has diverged both structurally and functionally, having effectively lost the original function of $\mathrm{Ub}$ 

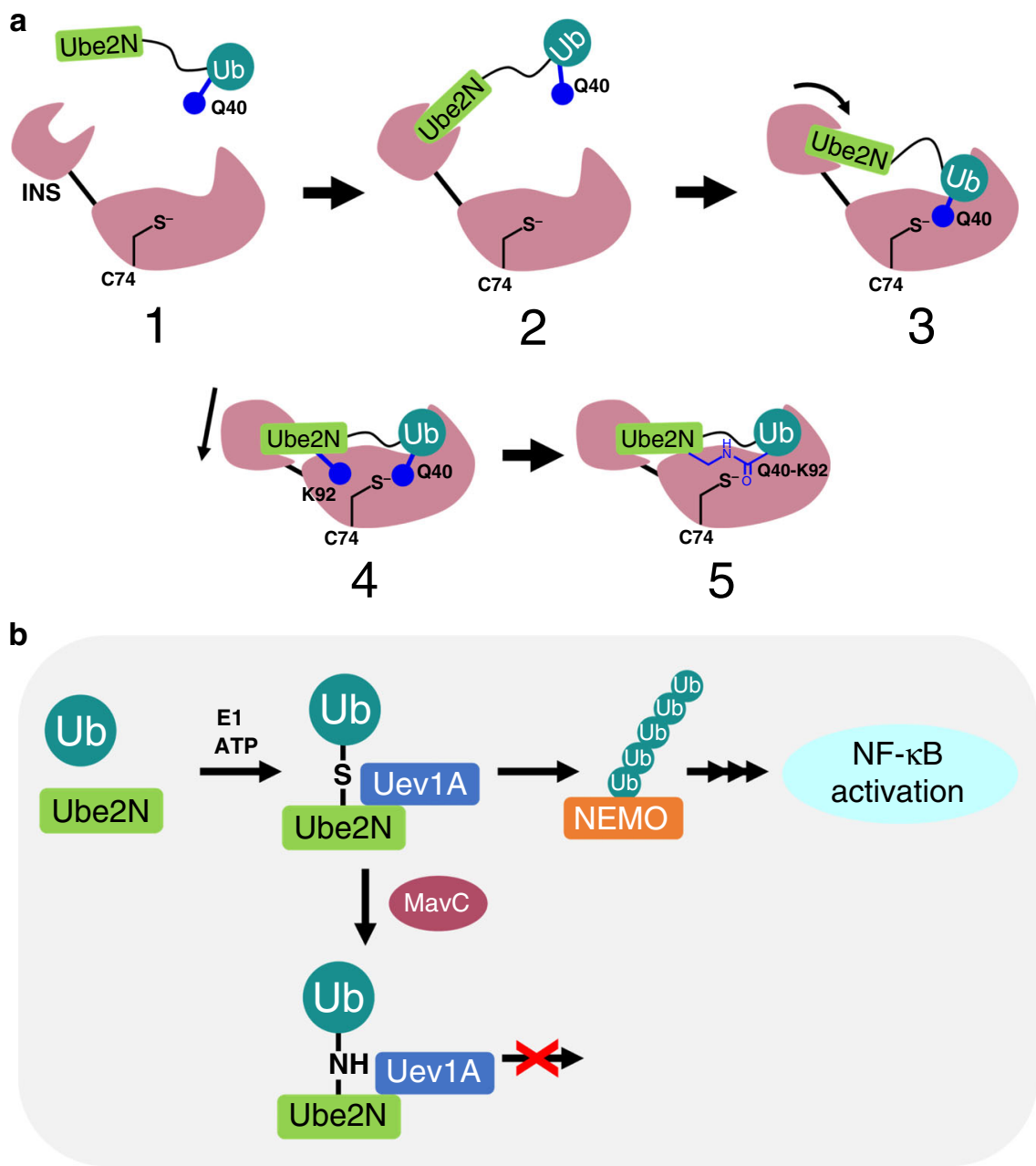

Fig. 7 Overall scheme of MavC-catalyzed ubiquitination. a Cartoon representation of Ube2N-Ub binding to MavC (1). INS domain of initially captures Ube2N moiety of substrate (2), moves to a rotated position where Ub has bound in the active site (3), and further adjusts to position Ube2 $\mathrm{N}$ for catalysis (4). b Scheme of MavC ubiquitination and the consequences thereof. $\mathrm{K} 63$ chain formation is prevented by ubiquitination of Ube2N, resulting in a dampening of the immune response.

and NEDD8 deamidation through a lower Ub affinity. Instead, it preferentially attacks the Ube2N-Ub conjugate to turn off Ube2N's ability to generate K63 poly-Ub chains. Despite both free $\mathrm{Ub} / \mathrm{NEDD} 8$ deamidation and Ube2N ubiquitination ultimately being shown to lead to decrease in NF- $\mathrm{BB}$ activation, we speculate that the divergence of MavC occurred to accommodate other $L$. pneumophila effectors that utilize the host's free Ub such as the E3 ligases LegU1 and SidC 36,37 and the noncanonical ligases of the SidE family. Poisoning of the host cell's supply of Ub by deamidation could antagonize these other effectors' activity. Therefore, MavC may satisfy a need for an alternative method of attenuating host immune signaling without perturbing the free $\mathrm{Ub}$ pool. How hampering the host's ability to make K63 poly-Ub chains may lead to other cellular effects remains to be elucidated.

\section{Methods}

Cloning, expression, and purification of recombinant proteins. $\mathrm{MavC}_{1-384}$ was PCR amplified from a plasmid encoding full-length MavC or MavC C74A using a PCR premix kit (Bioneer) cloned into pGEX-6p-1 plasmid (GE Healthcare). The resulting expression plasmid was transformed into BL21(DE3) strain of E. coli (Novagen). Cells were grown in $\mathrm{LB}$ media at $37^{\circ} \mathrm{C}$ to an $\mathrm{OD}_{600}$ of 0.6 , cooled to $18^{\circ} \mathrm{C}$, and induced overnight at the reduced temperature by addition of $0.35 \mathrm{mM}$ isopropyl-1-thio- $\beta$-D-galactopyranoside (IPTG). Cells obtained from $6 \mathrm{~L}$ bacterial culture were resuspended in phosphate-buffered saline (PBS) buffer ( $\mathrm{pH} 7.4$ ) supplemented with $0.4 \mathrm{M} \mathrm{KCl}$ (GST binding buffer). Resuspended cells were disrupted under high pressure using a French press. The lysate was centrifuged for an hour at $100,000 \times g$ at $4^{\circ} \mathrm{C}$, the supernatant was passed through a self-packed column of $5 \mathrm{~mL}$ glutathione-Sepharose resin (GE Healthcare) pre-equilibrated with GST-binding buffer. Following this, the resin was washed with five column volumes of GST-binding buffer. The bound fusion proteins were eluted with GST elution buffer $(250 \mathrm{mM}$ Tris $\mathrm{pH} 8,500 \mathrm{mM} \mathrm{KCl}$, and $10 \mathrm{mM}$ reduced glutathione) The protein eluted off the column was dialyzed against two changes $(4 \mathrm{~L})$ of dialysis buffer ( $1 \times$ PBS supplemented with $0.5 \mathrm{M} \mathrm{KCl}$ and $1 \mathrm{mM} \mathrm{DTT}$ ) at $4{ }^{\circ} \mathrm{C}$ to remove excess glutathione while being incubated with GST-tagged PreScission Protease as per the manufacturer's recommendation (GE Healthcare). The dialyzed sample was again passed through $5 \mathrm{~mL}$ of pre-equilibrated GST column to capture both free GST tag and the GST-tagged protease. The pure GST tag-cleaved protein, collected in the flow through, was concentrated to $44 \mathrm{mg} / \mathrm{mL}$ and buffer-exchanged into protein storage buffer ( $50 \mathrm{mM}$ Tris $\mathrm{pH} 7.4,50 \mathrm{mM} \mathrm{NaCl}$ and $1 \mathrm{mM}$ DTT). The purity and the homogeneity of protein samples from every stage of expression and purification were monitored by sodium dodecyl sulfate polyacrylamide gel electrophoresis (SDS-PAGE). MavC-INS (residues 128-225), MavC ${ }_{1-462}, \mathrm{MavC}_{8-400}$, all of the MavC mutants used in this study, E1, Ubc13, Ube2R1, Ube2S, and Uev1A were also expressed and purified similarly. E1, Ubc13, Ube2R1, and Ube2S constructs were obtained from Genentech, and Uev1A was obtained from Yusuke Sato (University of Tokyo).

Ube2N (full-length) cloned into pET-SUMO was purchased from Addgene (Plasmid \#51131). The plasmid, transformed into BL21(DE3) strain of E. coli, was expressed, lysed, and centrifuged same as the $\mathrm{MavC}$ constructs. The supernatant was passed through a pre-packed $5 \mathrm{~mL}$ HisTrap column (GE Healthcare) preequilibrated with binding buffer. Once the supernatant was loaded the resin was washed with five column volumes of binding buffer to wash off any unbound protein. This was followed by a $50 \mathrm{mM}$ imidazole wash (binding buffer supplemented with $50 \mathrm{mM}$ imidazole). The bound fusion protein was eluted with 
elution buffer ( $1 \times$ PBS supplemented with $300 \mathrm{mM}$ imidazole). The protein eluted off the HisTrap column was dialyzed against two changes $(4 \mathrm{~L})$ of dialysis buffer (1× PBS supplemented with $0.5 \mathrm{M} \mathrm{KCl}$ ) at $4{ }^{\circ} \mathrm{C}$ to remove excess imidazole while being incubated with His-tagged SUMO protease (SENP2, purified via $\mathrm{Ni}^{2}$ ${ }^{+}$-affinity chromatography method described herein). The dialyzed sample was again passed through a pre-equilibrated $5 \mathrm{~mL}$ HisTrap column to capture both the tag and the protease. The tag-cleaved protein, collected in the flow through, was concentrated to $9.3 \mathrm{mM}$ and buffer-exchanged into protein storage buffer $(50 \mathrm{mM}$ Tris $\mathrm{pH} 7.4,50 \mathrm{mM} \mathrm{NaCl}$, and $1 \mathrm{mM}$ DTT).

Cif from Yersinia pseudotuberculosis was cloned into pQE30 vector, expressed, and purified similarly to Ube2N, with the exception of the tag cleavage step.

$\mathrm{Ub}$ constructs were cloned into the pRSET-A vector and transformed into the BL21(DE3) strain of E. coli. Protein expression was carried out as above. After cell disruption, lysates were subjected to heating at $70-80^{\circ} \mathrm{C}$ for $15 \mathrm{~min}$ before ultracentrifugation, carried out as described above. Supernatant was adjusted to $\mathrm{pH}$ 4.5 with buffer containing $50 \mathrm{mM}$ sodium acetate and spun down to remove precipitates, if necessary. The cleared supernatant was loaded onto a self-packed column of SP Sepharose Fast Flow resin (GE Healthcare) and eluted with a gradient of $\mathrm{NaCl}$, up to $1 \mathrm{M}$. Fractions containing pure $\mathrm{Ub}$, confirmed by SDS-PAGE analysis, were pooled, concentrated, and exchanged into protein storage buffer (50 $\mathrm{mM}$ Tris $\mathrm{pH} 7.4,50 \mathrm{mM} \mathrm{NaCl}$, and $1 \mathrm{mM}$ DTT). The purity and the homogeneity of protein samples from every stage of expression and purification were monitored by SDS-PAGE.

Ube2N-SS-Ub, the disulfide conjugate, was prepared using a chemical method described by Lorenz et al..$^{38}$. To prepare the conjugate, C-terminal Gly76 ${ }^{\mathrm{Ub}}$ was first mutated to a cysteine by means of site-directed mutagenesis. The sequenceverified mutant was expressed and purified the same as the wild-type protein. The purified mutant, G76C $\mathrm{Cb}^{\mathrm{b}}$, was first activated by mixing it with DTNB (5,5'-dithio-

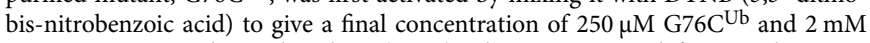
DTNB in $0.1 \mathrm{M}$ sodium phosphate ( $\mathrm{pH} 8)$. The reaction was left to incubate overnight at $4{ }^{\circ} \mathrm{C}$ to form thiol-nitrobenzene labeled Ub-mutant, TNB-G76 $\mathrm{C}^{\mathrm{Ub}}$. Excess DTNB was removed by buffer-exchanging the protein into assay buffer (50 $\mathrm{mM}$ Tris, $100 \mathrm{mM} \mathrm{NaCl}$ at $\mathrm{pH}$ 8). The disulfide conjugate was prepared by mixing equimolar ratio of Ube2N and TNB-G76 $\mathrm{C}^{\mathrm{Ub}}$ in the assay buffer. The reaction was left to incubate for $3 \mathrm{~h}$ at $25^{\circ} \mathrm{C}$ (with gentle rocking). The disulfide-linked protein complex, Ube2N-SS-Ub, was isolated by size-exclusion chromatography (Superdex 75 ) in the assay buffer. Fractions containing pure conjugate were pooled and concentrated to $\sim 7 \mathrm{mg} / \mathrm{mL}$.

To generate the ubiquitinated Ube $2 \mathrm{~N}$ product, purified $\mathrm{MavC}_{1-462}$ was combined with Ube $2 \mathrm{~N}$ and $\mathrm{Ub}$ at a final concentration of $1 \mu \mathrm{M} \mathrm{MavC}, 25 \mu \mathrm{M}$ Ube2N, and $100 \mu \mathrm{M}$ Ub in a final volume of $30 \mathrm{~mL}$. Reaction was allowed to proceed at $37^{\circ} \mathrm{C}$ for $3 \mathrm{~h}$ in $50 \mathrm{mM}$ Tris $\mathrm{pH} 7.4,100 \mathrm{mM} \mathrm{NaCl}$ and $1 \mathrm{mM}$ DTT, then concentrated to $1 \mathrm{~mL}$, and passed through two Superdex 200 Increase 10/300 GL columns (GE Healthcare), connected in tandem, in the reaction buffer described above. Fractions containing pure Ub-Ube2N were pooled and concentrated to approximately $10 \mathrm{mg} / \mathrm{mL}$.

Primers used for cloning the constructs in this study are listed in Supplementary Table 1.

\section{Complex formation, crystallization, data processing. Complex of C74A-}

$\mathrm{MavC}_{1-384}$ with Ube2N-SS-Ub was formed by mixing the two in a 1:1 molar ratio to give a final concentration of $32 \mathrm{mg} / \mathrm{mL}$ and incubating the mixture on ice for an hour. Crystals were grown by hanging drop vapor diffusion method at $21^{\circ} \mathrm{C}$. Crystals appeared in several conditions of the PEG-Ion screen from Hampton Research. The conditions identified from the commercial screens were optimized by reducing the protein concentration to $24 \mathrm{mg} / \mathrm{mL}$ during manual crystallizations. Data were collected from crystals grown in three different conditions. Crystal form $\mathrm{I}$, grown in $0.1 \mathrm{M}$ sodium acetate ( $\mathrm{pH} 4.6)$ and $3.5 \mathrm{M}$ sodium formate, diffracted to $1.97 \AA$ at the Advanced Photon Source (APS) at Argonne National Laboratories on the LS-CAT 21-ID-G $(\lambda=0.97 \AA)$ beam line. Data were processed and scaled using HKL2000 (ref. ${ }^{39}$ ) in the R3 space group with hexagonal setting, R3:H (Supplementary Table 2). Crystal form II, grown in $0.2 \mathrm{M}$ potassium bromide and $30 \%$ PEG 2000 MME, diffracted to $2.3 \AA$ at the Advanced Photon Source (APS) at Argonne National Laboratories on the LS-CAT 21-ID-G $(\lambda=0.97 \AA)$ beam line. Data were processed and scaled using HKL2000 (ref. ${ }^{39}$ ) in centered orthorhombic space group, C222 (Supplementary Table 2). Crystal form III, grown in $0.2 \mathrm{M}$ sodium formate and $20 \%$ PEG 3350, diffracted to $2.8 \AA$ at the Advanced Photon Source (APS) at Argonne National Laboratories on the LS-CAT 21-ID-G $(\lambda=0.97$ $\AA$ ) beam line. Data were processed and scaled using HKL2000 (ref. ${ }^{39}$ ) in hexagonal space group, $\mathrm{P}_{5}$ (Supplementary Table 2).

Purified $\mathrm{MavC}_{1-384}$ and Ub-Ube2N were mixed in a 1:1 molar ratio and incubated on ice for up to an hour. The resulting complex was concentrated to around $28 \mathrm{mg} / \mathrm{mL}$. Crystals were grown by hanging drop vapor diffusion method at $21^{\circ} \mathrm{C}$ in crystallization buffer containing 25\% PEG 3350 and $0.2 \mathrm{M}$ sodium malonate at $\mathrm{pH} 7.0$. To confirm the presence of the desired complex in the crystals, SDS-PAGE analysis was performed after washing crystals with crystallization buffer and dissolving in water. The best diffracting crystals grew in an optimized crystallization condition containing 25\% PEG 3350 and 0.2 M sodium malonate at pH 9.0 with $10 \mathrm{mM} \mathrm{NiCl}_{2}$. A complete dataset to $2.07 \AA$ was collected from a single crystal at the Advanced Photon Source (APS) at Argonne National Laboratories on the LS-CAT 21-ID-G $(\lambda=0.97857)$ beam line. Data were processed and scaled using HKL2000 (ref. ${ }^{39}$ ) in the hexagonal space group, $\mathrm{P}_{5}$ (Supplementary Table 2).

$\mathrm{MavC}_{\text {INS }}$ was crystallized using a solution containing a 1:2 molar ratio $(14 \mathrm{mg} /$ $\mathrm{mL}$ ) of $\mathrm{MavC}_{\mathrm{INS}}$ to Ube2N and incubated at overnight $4^{\circ} \mathrm{C}$. Crystals were grown by hanging drop diffusion method at $21^{\circ} \mathrm{C}$. Best diffracting crystals were obtained by streak seeding in a buffer containing $2 \mathrm{M}$ ammonium sulfate, $0.2 \mathrm{M}$ lithium sulfate, and $0.1 \mathrm{M}$ CAPS: $\mathrm{NaOH} ; \mathrm{pH} 10.5$. A complete dataset at $1.52 \AA$ was collected from a single crystal at the Advanced Photon Source (APS) at Argonne National Laboratories on the LS-CAT 21-ID-F $(\lambda=0.978772)$ beam line. Data were processed and scaled using HKL2000 (ref. ${ }^{39}$ ) in the trigonal space group, P3 (Supplementary Table 2).

Structure determination and refinement. The structures of $\mathrm{MavC}_{1-384}-\mathrm{Ube} 2 \mathrm{~N}-$ SS-Ub complex in three different space groups was determined by maximum likelihood molecular replacement using the program PHASER 40 from CCP4 suite ${ }^{41}$. The initial search models used were native $\mathrm{MavC}_{7-384}$ (PDB code $5 \mathrm{TSC}^{21}$ ), Ube2N (PDB code $2 \mathrm{C}^{2} \mathrm{~V}^{42}$ ), and Ub (PDB code $\left.1 \mathrm{UBQ}^{43}\right)$. The asymmetric unit consists of one complex in each of the three space groups. Iterative rounds of model building with the program $\mathrm{COOT}^{44}$ and refinement with the program PHENIX ${ }^{45}$ was used to arrive at the final structures that were validated through MolProbity ${ }^{46}$ and deposited with the Protein Data Bank.

The structure of the complex between $\mathrm{MavC}_{1-384}$ and Ube2N-SS-Ub in the $\mathrm{P}_{5}$ space group (early complex), determined at $2.80 \AA$ resolution (Supplementary Table 2), has one molecule of $\mathrm{MavC}_{1-384}$ engaging with one molecule of Ub-Ube $2 \mathrm{~N}$ in the asymmetric unit. Analysis of the Ramachandran plot ${ }^{47}$ indicated that $95.3 \%$ of residues fall in the most favored region and $4.7 \%$ in the additional allowed regions of the plot, none in the disallowed region of the plot. Electron density for most of the residues from MavC, Ub, and Ube2N are well resolved in the crystal structure. The final structure was validated with MolProbity ${ }^{46}$ and deposited in the Protein Data Bank (PDB code for the coordinate and the reflection files is 6UMP).

The structure of the complex between $\mathrm{MavC}_{1-384}$ and Ube2N-SS-Ub in the R3 space group (attacking complex I), determined at $1.97 \AA$ resolution (Supplementary Table 2), has one molecule of $\mathrm{MavC}_{1-384}$ engaging with one molecule of Ube2N-SS-Ub in the asymmetric unit. Analysis of the Ramachandran plot $^{47}$ indicated that $98.8 \%$ of residues fall in the most favored region and $1.2 \%$ in the additional allowed regions of the plot, none in the disallowed region of the plot. Electron density for almost all of the residues from MavC, Ub, and Ube2N are well resolved. Residues from 89 TO 94 in Ube2N have almost no density and hence have not been modeled in the structure. A total of 298 water molecules (an average B-factor of $42.6 \AA^{2}$ ) were observed in the asymmetric unit (PDB code for the coordinate and the reflection files is 6ULH).

The structure of the complex between $\mathrm{MavC}_{1-384}$ and Ube2N-SS-Ub in the C222 1 space group (attacking complex II), determined at $2.34 \AA$ resolution (Supplementary Table 2), has one molecule of $\mathrm{MavC}_{1-384}$ engaging with one molecule of Ube2N-SS-Ub in the asymmetric unit. Analysis of the Ramachandran plot $^{47}$ indicated that $97.8 \%$ of residues fall in the most favored region and $2.2 \%$ in the additional allowed regions of the plot, none in the disallowed region of the plot. Electron density for almost all of the residues from MavC, Ub, and Ube2N are well resolved. Residues from 89 to 94 in Ube2N have almost no density in this complex as well and hence have not been modeled in the structure. A total of 168 water molecules were observed in the asymmetric unit (PDB code for the coordinate and the reflection files is 6UMS).

The structure of $\mathrm{MavC}_{1-384}-\mathrm{Ub}-\mathrm{Ube} 2 \mathrm{~N}$ complex (product complex) was determined by maximum likelihood molecular replacement using the program $\mathrm{PHASER}^{40}$ from CCP4 suite ${ }^{41}$. The initial search models used were native $\mathrm{MavC}_{7}$ ${ }_{384}\left(\mathrm{PDB}\right.$ code $5 \mathrm{TSC}^{21}$ ), Ube2N (PDB code $2 \mathrm{C}^{2} \mathrm{~V}^{42}$ ), and Ub (PDB code $1 \mathrm{UBQ}^{43}$ ). The asymmetric unit consists of one complex. Iterative rounds of model building with the program COOT $^{44}$ and refinement with the program PHENIX ${ }^{45}$ resulted in the final structure for all data between 40 and $2.1 \AA$ resolution (Supplementary Table 2). The final structure was validated through MolProbity ${ }^{46}$ and deposited in the Protein Data Bank (PDB code for the coordinate and the reflection files is 6P5B). The structure of the complex between $\mathrm{MavC}_{1-384}$ and ubiquitinated $\mathrm{Ub}$ (Ub-Ube2N), determined at $2.07 \AA$ resolution (Supplementary Table 2), has one molecule of $\mathrm{MavC}_{1-384}$ engaging with one molecule of $\mathrm{Ub}-\mathrm{Ube} 2 \mathrm{~N}$ in the asymmetric unit. Analysis of the Ramachandran plot ${ }^{47}$ indicated that $97.9 \%$ of residues fall in the most favored region and $2.1 \%$ in the additional allowed regions of the plot, none in the disallowed region of the plot. Electron density for the mainchain of the $\mathrm{MavC}_{1-384}$ construct along with four non-native residues at the $\mathrm{N}$ terminal end remaining after the cleavage of the GST affinity tag are well resolved in the crystal structure. A total of 197 water molecules (an average B-factor of 43.4 $\AA^{2}$ ) were observed in the asymmetric unit. A separate model with the catalytic Cys of MavC included was generated using COOT.

The MavC insertion domain $\left(\mathrm{MavC}_{\mathrm{INS}}\right)$ structure was determined by maximum likelihood molecular replacement using the program PHASER ${ }^{40}$ in the PHENIX ${ }^{45}$ suite. The initial search model used was the insertion domain from $\mathrm{MavC}_{7-384}$ (PDB code: $5 \mathrm{TSC}^{21}$ ). The asymmetric unit contained two molecules, which were consistent with the formation of a crystallographic dimer. The structure was taken through multiple steps of model building with the program $\mathrm{COOT}^{44}$ and 
refinement with the program PHENIX ${ }^{45}$ which resulted in a final structure for all data between 29.8 and $1.53 \AA$ resolution (Supplementary Table 2). The final structure was validated through MolProbity 46 and deposited with the Protein Data Bank (PDB code for the coordinates and structure factors is $6 \mathrm{P} 5 \mathrm{H}$ ).

Residues where poor electron density was observed were modeled as either alanines or glycines. Interface area and residues at the interface in all the complexes presented in this study were computed using the web-based server, PISA, at the European Bioinformatics Institute (http://www.ebi.ac.uk/pdbe/prot_int/pistart. $\mathrm{html})^{48}$. All structures in this work were rendered and presented using Pymol (http://pymol.org)

Mutagenesis. Plasmids harboring the desired mutations and/or truncations of MavC were constructed via site-directed mutagenesis using mutagenic primer pairs. The resulting mutant PCR products were digested by $D p n I$ to remove the methylated templates and transformed into E. coli DH5a (home-made competent cells). Presence of desired mutations was confirmed by sequencing before being transformed into E. coli BL21(DE3) for protein expression and subsequent purification, similar to the aforementioned MavC constructs.

Primers used for mutagenesis in this study are listed in Supplementary Table 1.

GST pulldown assays. The pulldown assays were performed with GST-fusion proteins of $\mathrm{MavC}_{1-462}, \mathrm{MavC}_{\mathrm{INS}}$, and $\mathrm{MavC}_{\Delta \mathrm{INS}}$ as probe proteins to pulldown Ube2N. A $100 \mu \mathrm{L}$ of a $50 \%$ slurry of glutathione-agarose beads were equilibrated with $10 \times$ bed volume of $1 \times$ PBS buffer; $\mathrm{pH}$ 7.4. The beads were then centrifuged for $5 \mathrm{~min}$ at $5000 \mathrm{rpm}$ and the supernatant discarded. This wash step was repeated twice more. A total of $100 \mu \mathrm{L}$ of a $100 \mu \mathrm{M}$ stock of the probe protein(s) was used to charge the beads. These were then incubated at $4^{\circ} \mathrm{C}$ with end-over-end mixing for $4 \mathrm{~h}$, to ensure that the bait protein bound to the beads. The loaded beads were centrifuged for $5 \mathrm{~min}$ at $5000 \mathrm{rpm}$ and the flow through collected. The beads were washed as before and the wash collected after each step. Following the washes, the charged beads were incubated with $100 \mu \mathrm{L}$ of a $100 \mu \mathrm{M}$ stock of Ube2N. Binding was allowed to proceed overnight at $4{ }^{\circ} \mathrm{C}$ with end-over-end mixing. After incubation, the beads were centrifuged and washed as above, again collecting the flow through and wash at every step. Proteins were eluted by incubating the beads in 50 $\mu \mathrm{L}$ of GST elution buffer $(250 \mathrm{mM}$ Tris $\mathrm{pH} 8,500 \mathrm{mM} \mathrm{KCl}$, and $10 \mathrm{mM}$ reduced glutathione) for $15 \mathrm{~min}$ at room temperature followed by centrifugation. The elution step was repeated once more. The collected samples were analyzed by SDSPAGE.

Biolayer interferometry. Inactive (C74A) poly-His-tagged MavC constructs for use in the BLI studies were generated using two-step PCR (Megaprimer method) and verified using DNA sequencing. Primers used for cloning the constructs in this study are listed in Supplementary Table 1. The His-tagged proteins were expressed and purified as described above for Ube2N. Homogeneity of all the purified proteins was confirmed by SDS-PAGE analysis. The MavC constructs used for these studies were diluted in BLI buffer ( $1 \times$ PBS containing $0.05 \% \mathrm{v} / \mathrm{v}$ Tween -20 and $0.1 \% \mathrm{w} / \mathrm{v} \mathrm{BSA}$ ) to a concentration of $25 \mu \mathrm{g} / \mathrm{mL}$. The analytes were also diluted in the same buffer at the following concentrations: Ub $(2 \mathrm{mM})$, Ube2N $(100 \mu \mathrm{M})$, UbSS-Ube2N $(100 \mu \mathrm{M})$, and Ub-Ube2N $(100 \mu \mathrm{M})$. Serial dilutions of each analyte were prepared, in replicates of three, for analysis. One Ni-NTA biosensor was used for each $K_{\mathrm{D}}$ measurement, dipping the MavC protein loaded tip into wells that contained an analyte, starting with the lowest concentration of analyte first. The direct binding experiment was performed for $120 \mathrm{~s}$ for association and $100 \mathrm{~s}$ for dissociation in BLI buffer using a biolayer interferometer (BLI), Octet Red 96 system (Pall ForteBio, Corp., Menlo Park, CA, USA), and data acquired using the ForteBio Data Acquisition 8.2 software (Pall ForteBio Corp., Menlo Park, CA, USA). Association responses (from 110 to $115 \mathrm{~s}$ ) was averaged and plotted in BAL Octet Data Analysis Software. The data were fit to a non-linear regression one site- - specific binding model to determine the $K_{\mathrm{D}}$.

Protein expression, purification, and NMR spectroscopy. ${ }^{15} \mathrm{~N}$-labeled Ub and Ube2N were grown in minimal MOPS media supplemented with ${ }^{15} \mathrm{NH}_{4} \mathrm{Cl}$. ${ }^{2} \mathrm{H},{ }^{15} \mathrm{~N}$-labeled $\mathrm{Ub}_{\mathrm{WT}}, \mathrm{Ub}_{\mathrm{G} 76 \mathrm{C}}$, and Ube2 $\mathrm{N}_{\mathrm{WT}}$ were grown in minimal $\mathrm{M} 9$ media prepared in $\mathrm{D}_{2} \mathrm{O}$ and supplemented with ${ }^{15} \mathrm{NH}_{4} \mathrm{Cl}$. In all cases, Ub and Ube2N constructs were expressed and purified as previously described ${ }^{31}$. The disulfide Ube2N-SS-Ub conjugate was prepared as described above ${ }^{38}$. NMR data were collected at $25^{\circ} \mathrm{C}$ on a 500Mhz Bruker AVANCE III, or $600 \mathrm{MHz}$ AVANCEII fitted with a cryoprobe. NMR samples were prepared in $25 \mathrm{mM}$ Tris, $100 \mathrm{mM}$ $\mathrm{NaCl}, \mathrm{pH} 7.6$ with the addition of $5 \% \mathrm{D}_{2} \mathrm{O}$. Samples typically contained $150 \mathrm{mM}$ isotopically labeled protein and unlabeled WT or C74A-MavC ${ }_{1-384}$ at concentrations from 0 to $225 \mathrm{mM}$. Data were processed with NMRPipe ${ }^{49}$ and analyzed using NMRView $J^{50}$. The magnitude of chemical shift changes for specific resonances followed during NMR titration experiments were determined using the following equation:

$$
\delta=\left(\left[\delta_{H}^{2}+a \delta_{N}^{2}\right] / 2\right)^{1 / 2}
$$

where $\delta_{\mathrm{H}}$ and $\delta_{\mathrm{N}}$ are the ${ }^{1} \mathrm{H}$ and ${ }^{15} \mathrm{~N}$ chemical shift changes, respectively, and 0.14 was used for the scaling factor $a^{51}$.
Ubiquitination and deamidation assays. To analyze the ubiquitinating activity of MavC mutants, purified $\mathrm{MavC}_{1-462}$ constructs (wild type or mutants) were combined with Ube2N-SS-Ub at a final concentration of $0.005 \mu \mathrm{M} \mathrm{MavC}$ and $25 \mu \mathrm{M}$ Ube2N-SS-Ub, and incubated at $37^{\circ} \mathrm{C}$ for $30 \mathrm{~min}$ in reaction buffer $(50 \mathrm{mM}$ Tris $\mathrm{pH}$ 7.4, $100 \mathrm{mM} \mathrm{NaCl}$ ). The reaction products were analyzed by SDS-PAGE and visualized with Coomassie Blue. To analyze the quadruple mutants an compare them with other mutants, an increased incubation time of $60 \mathrm{~min}$ and a [MavC] of $0.05 \mu \mathrm{M}$ was utilized. On the other hand, to analyze the ubiquitinating activity of $\mathrm{MavC}_{\Delta \mathrm{INS}}$ and also the ubiquitinating activity of MavC against Ubc13, UbE2S, and UbE2R1, $0.5 \mu \mathrm{M}$ MavC, $25 \mu \mathrm{M}$ Ube2N, and an extended incubation time of $60 \mathrm{~min}$ was used, and the Ub concentration was increased to $100 \mu \mathrm{M}$ in an attempt to promote ubiquitination.

$\mathrm{Ub}$ deamidating assays were performed by combining purified $\mathrm{MavC}_{1-462}$ constructs (wild type or mutants) with $\mathrm{Ub}$ at a final concentration of $0.5 \mu \mathrm{M}$ enzyme and $100 \mu \mathrm{M} \mathrm{Ub}$. The reactions were incubated at $37^{\circ} \mathrm{C}$ for $30 \mathrm{~min}$ in reaction buffer (50 mM Tris pH 7.4, $100 \mathrm{mM} \mathrm{NaCl}, 1 \mathrm{mM}$ DTT). The deamidation reaction products were analyzed by Native-PAGE and visualized with Coomassie Blue.

MavC's deamidating activity on the disulfide conjugate was tested by combining purified $\mathrm{MavC}_{1-462}$ with Ube2N-SS-Ub at a final concentration of $0.005 \mu \mathrm{M}$ MavC and $25 \mu \mathrm{M}$ Ube2N-SS-Ub and incubated at $37^{\circ} \mathrm{C}$ for $30 \mathrm{~min}$ in reaction buffer $(50$ $\mathrm{mM}$ Tris $\mathrm{pH} 7.4,100 \mathrm{mM} \mathrm{NaCl}$ ). The reaction products were analyzed by NativePAGE and visualized with Coomassie Blue. As a control to observe the migration of deamidated Ube2N-SS-Ub, a reaction was run utilizing the known deamidase Cif at a concentration of $0.5 \mu \mathrm{M}$.

To determine the Michaelis-Menten kinetic parameters of the Ube2N ubiquitinating activity of MavC, reactions were conducted with MavC $(0.5 \mu \mathrm{M})$, and varying concentrations of Ube2 $\mathrm{N}$ at $37^{\circ} \mathrm{C}$ for $30 \mathrm{~min}$. Reactions were quenched with SDS-PAGE loading buffer and separated by SDS-PAGE along with Ube2N standards of known concentrations and visualized with Coomassie Blue. Gels were analyzed with ImageJ, and a standard curve was generated using the band intensities of the Ube2N standards. This standard curve was used to quantify Ube2N produced from each reaction. Data were fit to the Michaelis-Menten equation. Linear regression and plotting were performed using SigmaPlot. Reactions were performed in triplicate for kinetic analysis. Error bars were generated via the standard deviation.

To determine the Michaelis-Menten kinetic parameters of the Ub deamidating activity of MavC, reactions were conducted with MavC $(0.5 \mu \mathrm{M})$, and varying concentrations of $\mathrm{Ub}$ at $37^{\circ} \mathrm{C}$ for $30 \mathrm{~min}$. Reactions along with $\mathrm{Ub}$ standards of known concentrations were separated by Native-PAGE. Gels were analyzed by ImageJ as described above. All reactions were performed in triplicate. Error bars were generated via the standard deviation.

To determine the activity of MavC against the Uevla:Ube2N-SS-Ub complex, varying amounts of Uevla were incubated with Ube2N-SS-Ub for 10 min prior to addition of $0.005 \mu \mathrm{M}$ MavC. Reactions were performed at $37^{\circ} \mathrm{C}$ for $30 \mathrm{~min}$. Reactions were quenched with SDS-PAGE loading buffer, separated by SDS-PAGE, and visualized with Coomassie Blue.

MavC-mediated ubiquitination of Ube2N during $L$. pneumophila infection. $L$. pneumophila strains were grown to post-exponential phase $\left(\mathrm{OD}_{600}=3.2-3.8\right)$ in AYE medium at $37^{\circ} \mathrm{C}$ and then induced for $4 \mathrm{~h}$ with $0.2 \mathrm{mM}$ IPTG before infection. L. pneumophila strains were obtained from prior studies ${ }^{22,52}$. Raw 264.7 cells or U937 cells were infected with L. pneumophila strains at an MOI of 10 for $2 \mathrm{~h}$. Cells were washed three times with PBS and then lysed with $0.2 \%$ saponin on ice for $30 \mathrm{~min}$. Cell lysates were resolved by SDS-PAGE and probed with MavC (1:5000 dilution) ${ }^{22}$-specific and Ube2N (1:1000 dilution)-specific antibodies. Anti-Ube2N antibody was purchased from Thermo Fisher Scientific (catalog \# 37-1100). Tubulin and ICDH were used as a loading controls and probed using anti-tubulin antibody (1:10,000 dilution) from DSHB (catalog \# E7) and antiICDH $(1: 10,000)^{53}$

E1 charging assay. To compare the ability of E1 to charge Ube2N versus Ub-Ube2N, a reaction mixture of $0.5 \mu \mathrm{M}$ E1 enzyme, $200 \mu \mathrm{M}$ Ube2N or Ub-Ube2N, $400 \mu \mathrm{M}$ Ub was conducted in a reaction buffer consisting of $50 \mathrm{mM}$ Tris pH 7.4, $100 \mathrm{mM} \mathrm{NaCl}, 2.5 \mathrm{mM} \mathrm{ATP}, 5 \mathrm{mM} \mathrm{MgCl}_{2}$. Reactions were allowed to proceed for $30 \mathrm{~min}$ at $37^{\circ} \mathrm{C}$ before quenching with either reducing or nonreducing SDS-PAGE buffer, separated by SDS-PAGE and visualized with Coomassie Blue.

NF-kB activation assay. HEK293T cells were grown to $70 \%$ confluence in 24 -well plates, and transfected with $100 \mathrm{ng}$ NF- $\kappa B$ reporter plasmids and $10 \mathrm{ng}$ of plasmid that directs the expression of Renilla luciferase in pRL-SV40 (Promega) as internal control. Four hundred nanograms $4 x F l a g-M a v C$ vector or its mutant and $400 \mathrm{ng}$ 4xFlag-TRAF6 were co-transfected at the same time. After $24 \mathrm{~h}$, the cells were then collected and lysed for NF-kB luciferase reporter assay following the manufacturer's protocols (Promega cat. no. E1910). Briefly, cells were lysed with $100 \mu \mathrm{L}$ passive lysis and $20 \mu \mathrm{L}$ of cell lysates transferred to a 96-well plate. Dispense $100 \mu \mathrm{L}$ Luciferase assay buffer containing Firefly luciferase substrate and measure Firefly luciferase activity. After that, $100 \mu \mathrm{L}$ Luciferase Stop and Glo reagent was added 
and Renilla luciferase activity measured. The expression of MavC or its mutant was probed in lysates of transfected cells and the blots shown are representatives of at least three independent experiments. Anti-MavC antibody ${ }^{22}$ was used at a dilution of 1:5000. Tubulin was used as a loading control and probed using anti-tubulin antibody (1:10,000 dilution) from DSHB (catalog \# E7).

Statistical methods. The gels presented in this study are representative of three different experiments performed independently, with similar results obtained (Figs. 2e, 4c, d, 5d, e, Supplementary Figs. 1d, 2k, 3a-c, e, 4c and 5a, b, e). No statistical method was used to predetermine sample size. The experiments were not randomized and were not performed with blinding to the conditions of the experiments.

Reporting summary. Further information on research design is available in the Nature Research Reporting Summary linked to this article.

\section{Data availability}

Coordinates of all five structures have been deposited into the Protein Data Bank under accession codes $6 \mathrm{UMP}, 6 \mathrm{ULH}, 6 \mathrm{UMS}, 6 \mathrm{P} 5 \mathrm{~B}$ and $6 \mathrm{P} 5 \mathrm{H}$. The source data underlying Figs. 2a, b, e, 4c, d, 5d, f, and Supplementary Figs. 1e, 2a, b, 3a, c, 3e, 4b, c, and 5a, b are provided as a Source Data file. Other data are available from the corresponding authors upon reasonable request.

Received: 24 October 2019; Accepted: 14 April 2020;

Published online: 12 May 2020

\section{References}

1. Komander, D. \& Rape, M. The ubiquitin code. Annu. Rev. Biochem. https:// doi.org/10.1146/annurev-biochem-060310-170328 (2012).

2. Hershko, A. et al. Components of ubiquitin-protein ligase system. J. Biol. Chem. 258, 8206-8214 (1983).

3. Ebner, P., Versteeg, G. A. \& Ikeda, F. Ubiquitin enzymes in the regulation of immune responses. Crit. Rev. Biochem. Mol. Biol. https://doi.org/10.1080/ 10409238.2017.1325829 (2017).

4. Grabbe, C., Husnjak, K. \& Dikic, I. The spatial and temporal organization of ubiquitin networks. Nat. Rev. Mol. Cell Biol. https://doi.org/10.1038/nrm3099 (2011).

5. Bhavsar, A. P., Guttman, J. A. \& Finlay, B. B. Manipulation of host-cell pathways by bacterial pathogens. Nature https:/doi.org/10.1038/nature06247 (2007).

6. Angot, A., Vergunst, A., Genin, S. \& Peeters, N. Exploitation of eukaryotic ubiquitin signaling pathways by effectors translocated by bacterial type III and type IV secretion systems. PLoS Pathogens https://doi.org/10.1371/journal. ppat.0030003 (2007).

7. Huibregtse, J. \& Rohde, J. R. Hell's BELs: bacterial E3 ligases that exploit the eukaryotic ubiquitin machinery. PLoS Pathog. https://doi.org/10.1371/journal. ppat.1004255 (2014).

8. Pruneda, J. N. et al. The molecular basis for ubiquitin and ubiquitin-like specificities in bacterial effector proteases. Mol. Cell 63, 261-276 (2016).

9. Sheedlo, M. J. et al. Structural basis of substrate recognition by a bacterial deubiquitinase important for dynamics of phagosome ubiquitination. Proc. Natl. Acad. Sci. USA 112, 15090-15095 (2015).

10. Sanada, T. et al. The Shigella flexneri effector OspI deamidates UBC13 to dampen the inflammatory response. Nature https://doi.org/10.1038/ nature10894 (2012).

11. Fu, P. et al. Complex structure of OspI and Ubc13: the molecular basis of Ubc13 deamidation and convergence of bacterial and host E2 recognition. PLoS Pathog. https://doi.org/10.1371/journal.ppat.1003322 (2013).

12. Song, L. \& Luo, Z.-Q. Post-translational regulation of ubiquitin signaling. J. Cell Biol. 218, 1776-1786 (2019).

13. Cui, J. et al. Glutamine deamidation and dysfunction of ubiquitin/NEDD8 induced by a bacterial effector family. Science https://doi.org/10.1126/ science.1193844 (2010).

14. Bhogaraju, S. et al. Phosphoribosylation of ubiquitin promotes serine ubiquitination and impairs conventional ubiquitination. Cell 167, 1636-1649. e13 (2016).

15. Qiu, J. et al. Ubiquitination independent of E1 and E2 enzymes by bacterial effectors. Nature 533, 120-124 (2016).

16. Kotewicz, K. M. et al. A single Legionella effector catalyzes a multistep ubiquitination pathway to rearrange tubular endoplasmic reticulum for replication. Cell Host Microbe 21, 169-181 (2017).

17. Puvar, K., Luo, Z. Q. \& Das, C. Uncovering the structural basis of a new twist in protein ubiquitination. Trends Biochem. Sci. https://doi.org/10.1016/j. tibs.2018.11.006 (2019)
18. Luo, Z.-Q. \& Isberg, R. R. Multiple substrates of the Legionella pneumophila Dot/Icm system identified by interbacterial protein transfer. Proc. Natl. Acad. Sci. USA https://doi.org/10.1073/pnas.0304916101 (2004).

19. Burstein, D. et al. Genomic analysis of 38 Legionella species identifies large and diverse effector repertoires. Nat. Genet. 48, 167-175 (2016).

20. Qiu, J. \& Luo, Z.-Q. Legionella and Coxiella effectors: strength in diversity and activity. Nat. Rev. Microbiol. 15, 591-605 (2017).

21. Valleau, D. et al. Discovery of ubiquitin deamidases in the pathogenic arsenal of Legionella pneumophila. Cell Rep. 23, 568-583 (2018).

22. Gan, N., Nakayasu, E. S., Hollenbeck, P. J. \& Luo, Z.-Q. Legionella pneumophila inhibits immune signalling via MavC-mediated transglutaminase-induced ubiquitination of UBE2N. Nat. Microbiol. 4, 134 (2019).

23. Yao, Q. et al. A bacterial type III effector family uses the papain-like hydrolytic activity to arrest the host cell cycle. Proc. Natl. Acad. Sci. USA https://doi.org/ 10.1073/pnas.0900212106 (2009).

24. Yao, Q. et al. Structural mechanism of ubiquitin and NEDD8 deamidation catalyzed by bacterial effectors that induce macrophage-specific apoptosis. Proc. Natl. Acad. Sci. USA https://doi.org/10.1073/pnas.1210831109 (2012).

25. Hodge, C. D., Spyracopoulos, L. \& Glove, J. N. M. Ubc13: the Lys63 ubiquitin chain building machine. Oncotarget https://doi.org/10.18632/oncotarget.10948 (2016).

26. Gan, N. et al. Legionella pneumophila regulates the activity of UBE2N by deamidase-mediated deubiquitination. EMBO J. 39, e102806 (2020).

27. Siepmann, T. J., Bohnsack, R. N., Tokgöz, Z., Baboshina, O. V. \& Haas, A. L. Protein interactions within the $\mathrm{N}$-end rule ubiquitin ligation pathway. J. Biol. Chem. 278, 9448-9457 (2003).

28. Nishide, A. et al. Structural basis for the recognition of Ubc13 by the Shigella flexneri effector OspI. J. Mol. Biol. 425, 2623-2631 (2013).

29. Stewart, M. D., Ritterhoff, T., Klevit, R. E. \& Brzovic, P. S. E2 enzymes: more than just middle men. Cell Res. https://doi.org/10.1038/cr.2016.35 (2016).

30. Merkley, N., Barber, K. R. \& Shaw, G. S. Ubiquitin manipulation by an E2 conjugating enzyme using a novel covalent intermediate. J. Biol. Chem. https:// doi.org/10.1074/jbc.M505205200 (2005).

31. Pruneda, J. N., Stoll, K. E., Bolton, L. J., Brzovic, P. S. \& Klevit, R. E. Ubiquitin in motion: structural studies of the ubiquitin-conjugating enzyme ubiquitin conjugate. Biochemistry 50, 1624-1633 (2011).

32. Bhattacharyya, R. \& Chakrabarti, P. Stereospecific interactions of proline residues in protein structures and complexes. J. Mol. Biol. 331, 925-940 (2003).

33. VanDemark, A. P., Hofmann, R. M., Tsui, C., Pickart, C. M. \& Wolberger, C. Molecular insights into polyubiquitin chain assembly: crystal structure of the Mms2/Ubc13 heterodimer. Cell 105, 711-720 (2001).

34. Clague, M. J., Heride, C. \& Urbé, S. The demographics of the ubiquitin system. Trends Cell Biol. 25, 417-426 (2015).

35. Eddins, M. J., Carlile, C. M., Gomez, K. M., Pickart, C. M. \& Wolberger, C. Mms2-Ubc13 covalently bound to ubiquitin reveals the structural basis of linkage-specific polyubiquitin chain formation. Nat. Struct. Mol. Biol. 13, 915-920 (2006).

36. Hsu, F. S. et al. The Legionella effector SidC defines a unique family of ubiquitin ligases important for bacterial phagosomal remodeling. Proc. Natl. Acad. Sci. USA https://doi.org/10.1073/pnas.1402605111 (2014).

37. Qiu, J. \& Luo, Z.-Q. Hijacking of the host ubiquitin network by Legionella pneumophila. Front. Cell. Infect. Microbiol. 7, 487 (2017).

38. Lorenz, S., Bhattacharyya, M., Feiler, C., Rape, M. \& Kuriyan, J. Crystal structure of a Ube2S-ubiquitin conjugate. PLOS ONE https://doi.org/10.1371/ journal.pone.0147550 (2016).

39. Otwinowski, Z. \& Minor, W. Processing of X-ray diffraction data collected in oscillation mode. Methods Enzymol. 276, 307-326 (1997).

40. McCoy, A. J. et al. Phaser crystallographic software. J. Appl. Crystallogr. 40, 658-674 (2007).

41. Collaborative Computational Project, Number 4. The CCP4 suite: programs for protein crystallography. Acta Crystallogr. D Biol. Crystallogr. 50, 760-763 (1994).

42. Zhang, M. et al. Chaperoned ubiquitylation-crystal structures of the CHIP U box E3 ubiquitin ligase and a CHIP-Ubc13-Uevla complex. Mol. Cell 20, 525-538 (2005).

43. Vijay-Kumar, S., Bugg, C. E. \& Cook, W. J. Structure of ubiquitin refined at 1.8 A resolution. J. Mol. Biol. 194, 531-544 (1987).

44. Emsley, P., Lohkamp, B., Scott, W. G. \& Cowtan, K. Features and development of Coot. Acta Crystallogr. D Biol. Crystallogr. 66, 486-501 (2010).

45. Adams, P. D. et al. PHENIX: a comprehensive python-based system for macromolecular structure solution. Acta Crystallogr. D Biol. Crystallogr. 66, 213-221 (2010).

46. Chen, V. B. et al. MolProbity: all-atom structure validation for macromolecular crystallography. Acta Crystallogr. D Biol. Crystallogr. 66, $12-21(2010)$. 
47. Ramachandran, G. N., Ramakrishnan, C. \& Sasisekharan, V. Stereochemistry of polypeptide chain configurations. J. Mol. Biol. 7, 95-99 (1963).

48. Krissinel, E. \& Henrick, K. Inference of macromolecular sssemblies from vrystalline state. J. Mol. Biol. https://doi.org/10.1016/j.jmb.2007.05.022 (2007).

49. Delaglio, F. et al. NMRPipe: a multidimensional spectral processing system based on UNIX pipes. J. Biomol. NMR 6, 277-293 (1995).

50. Johnson, B. A. \& Blevins, R. A. NMR View: a computer program for the visualization and analysis of NMR data. J. Biomol. NMR 4, 603-614 (1994)

51. Williamson, M. P. Using chemical shift perturbation to characterise ligand binding. Prog. Nucl. Magn. Reson. Spectrosc. 73, 1-16 (2013).

52. Berger, K. H. \& Isberg, R. R. Two distinct defects in intracellular growth complemented by a single genetic locus in Legionella pneumophila. Mol. Microbiol. 7, 7-19 (1993).

53. $\mathrm{Xu}, \mathrm{L}$. et al. Inhibition of host vacuolar $\mathrm{H}+$-ATPase activity by a Legionella pneumophila effector. PLOS Pathog. 6, e1000822 (2010).

\section{Acknowledgements}

This work was funded by National Institute of Health Grants R01GM126296 (to C.D.), T32GM132024 (to S.K.), R01AI127465 (to Z-Q.L.), and R01 GM088055 (to R.E.K.). We thank our staff contacts, Monica Green and Joe Brunzelle, on the LS-CAT beamlines, 21ID-F and 21-ID-G, at the Advanced Photon Source (Argonne National Laboratory) for their support during X-ray data collection. We thank Ronald Stenkamp for help comparing Ube $2 \mathrm{~N}$ structures. We acknowledge Genentech, Inc. for the gifts of the E1, Ubiquitin, Ube2R1, and Ube2S plasmids, and Yusuke Sato (University of Tokyo) for the UevlA plasmid. The contents of this publication are solely the responsibility of the authors and do not necessarily represent the official views of NIH.

\section{Author contributions}

K.P., S.I., Z-Q.L., P.S.B., R.E.K., and C.D. designed and interpreted experiments. P.S.B. and R.E.K. planned and carried out NMR analyses and designed biochemical assays. J.F. and ZQ.L. performed Legionella infection and MavC transfection experiments. S.K. performed BLI analyses. K.I.N.T. performed pulldown experiments. K.P and S.I. performed all other experiments including crystallization and structure determination of the protein complexes. K.P., S.I., P.S.B., R.E.K., and C.D. wrote the paper with editorial input from all authors.

\section{Competing interests}

The authors declare no competing interests.

\section{Additional information}

Supplementary information is available for this paper at https://doi.org/10.1038/s41467020-16211-x.

Correspondence and requests for materials should be addressed to R.E.K. or C.D.

Peer review information Nature Communications thanks Yongqun Zhu and the other, anonymous, reviewer(s) for their contribution to the peer review of this work. Peer reviewer reports are available.

Reprints and permission information is available at http://www.nature.com/reprints

Publisher's note Springer Nature remains neutral with regard to jurisdictional claims in published maps and institutional affiliations.

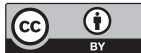

Open Access This article is licensed under a Creative Commons Attribution 4.0 International License, which permits use, sharing, adaptation, distribution and reproduction in any medium or format, as long as you give appropriate credit to the original author(s) and the source, provide a link to the Creative Commons license, and indicate if changes were made. The images or other third party material in this article are included in the article's Creative Commons license, unless indicated otherwise in a credit line to the material. If material is not included in the article's Creative Commons license and your intended use is not permitted by statutory regulation or exceeds the permitted use, you will need to obtain permission directly from the copyright holder. To view a copy of this license, visit http://creativecommons.org/ licenses/by/4.0/.

(C) The Author(s) 2020 